\title{
Oscillatory Magnetopolar Free Convection Flow Through a Vertical Porous Plate Embedded in a Porous Medium in Slip Flow Regime
}

\author{
N.C. Jain ${ }^{1}$, D. Chaudhary ${ }^{2}$ and R.N. Jat ${ }^{3}$ \\ Department of Mathematics, University of Rajasthan, Jaipur 302055, India
}

\begin{abstract}
In this paper we study as Oscillatory two dimensional magnetopolar free convection flow through a porous medium with combined heat and mass transfer and thermal radiation in slip flow regime. The permeability and suction velocity are assumed to be time dependent. Using perturbation technique expressions for velocity $(u)$, angular velocity $(\square)$, temperature $(\square)$, concentration $(C)$, skin friction $(\varnothing)$ and Nusselt number $(\mathrm{Nu})$ are obtained and a comparative study is made to analyze the effects of different parameters. We notice that as we increase permeability parameter $(K)$ skin friction falls in the beginning but rises as we move away from the plate. Moreover, for both the basic fluids air $(\operatorname{Pr}=0.71, S c=0.22)$ and water $(\operatorname{Pr}=7, S c=0.61)$, velocity increases on decreasing the slip at the boundary.
\end{abstract}

Key Words: Heat flux, Mass flux, Polar fluid, Radiation, Unsteady.

\section{Introduction}

The natural or free convection process is present in various physical phenomenon such as fire engineering, nuclear energy, fiber and granular insulation, geothermal system etc. Simultaneously, heat and mass transfer from different geometrics embedded in porous media have many engineering and geophysical applications such as geothermal reservoirs, drying of porous solids, thermal insulation, enhanced oil recovery etc. Magnetohydrodynamics has attracted the attention of a large number of scholars due to its diverse applications. In engineering it finds its applications in MHD pumps, MHD bearings etc. The phenomenon of mass transfer is also very common in theory of stellar structure and observable effects are detectable, at least on the solar surface. The study of effects of magnetic field on free convection flow is important in liquid metals, electrolyte and ionized gases.

Das and Das [1] have studied MHD free convection flow near a moving plate in presence of thermal radiation where as MHD free convection flow of a viscous fluid through a porous medium bounded by an oscillating plate was studied by Singh and Gupta [2]. Moreover, Ahmed [3] observed the effects of unsteady free convection MHD flow through a porous medium while Sahoo et al. [4] studied MHD unsteady free convection flow with constant suction and heat sink. Cookey et al. [5] studied the influence of viscous dissipation and radiation on unsteady MHD free convection flow in a porous medium with time dependent suction on the other hand Aboeldahab and Azzam [6] made studies on unsteady three dimensional combined heat and mass transfer with time dependent chemical reaction.

Convection heat transfer through a porous medium has been a subject of great interest for the last few decades as these are quite prevalent in nature.

Such flows have many engineering applications in petroleum technology to study the movement of natural gas, oil and water through oil reservoirs, in chemical engineering for filteration and purification process etc. In view of these applications, many scholars have made investigations where porous medium is either bounded by horizontal or vertical surface. Magyari et al. [7] found an analytic solution for unsteady free convection in porous media where as Geindreau and Auriault [8] studied MHD flows in porous media. Ahmed and Ahmed [9, 10] studied oscillatory two dimensional as well as three dimensional flows through a porous medium with viscous dissipative heat.

The Navier-stokes model of classical hydrodynamics has drastic limitations. It cannot describe fluids with microstructure, fluids that are interesting in themselves and important in application. In general, individual particles of such complex fluids, eg. Polymeric suspensions, animal blood, liquid crystals etc., may be of different shapes, may shrink and expand, or change their shape and moreover they may rotate independently of the rotation and movement of the fluid. Such fluids are called Polar fluids which belong to the class of fluids with nonsymmetric stress tensor. These fluids are more general than that considered by classical hydrodynamics that we call ordinary fluids \{Lukaszewicz [11]\}. Kim [12] studied unsteady MHD convection flow of a polar fluid in a porous medium Patil and Kulkarni [13] studied effects of chemical reaction on free convection flow of a polar fluid. Jain and Gupta $[14,15]$ studied effects of rotational parameter on unsteady magnetopolar free convection 
with different boundary conditions while Cheng [16] studied natural convection heat and mass transfer from a sphere in micropolar fluids.

It is a well known fact that in case of many polymeric liquids when the weight of the molecule is high, it shows slip at the boundary. In many problems like that of thin films, rarefied fluids, fluid containing concentrated suspension, the no slip boundary condition fails to work. Jain et al. [17] and Chaudhary and Jain [18] have studied effects of different parameters on combined heat and mass transfer in magneto polar fluid with slip flow regime. Fang et al. [19] studied slip MHD viscous flow over a stretching sheet while Sahin [20] studied the influence of chemical reaction on transient MHD free convective flow in slip flow regime.

In this paper we study an oscillatory magnetopolar free convection flow through a porous medium with time dependent permeability and suction velocity. The flow is also effected by rotational and couple stress parameters with combined heat and mass transfer and thermal radiation with slip flow regime. Using perturbation technique solutions have been obtained for velocity (u), angular velocity $(\square)$ temperature ( $\square$ ), concentration (C), skin friction $\left(\mathrm{C}_{\mathrm{f}}\right)$ and Nusselt number $(\mathrm{Nu})$ and are shown graphically for both the basic fluids air $(\mathrm{Pr}=0.71, \mathrm{Sc}=$ $0.22)$ and water $(\operatorname{Pr}=7, \mathrm{Sc}=0.61)$.

\section{Formulation of the problem}

An unsteady MHD free convective, two dimensional heat and mass transfer flow with radiation of a polar fluid through a porous medium in a slip flow regime with transverse magnetic field of strength $\mathrm{B}_{0}$ is considered. The plate is subjected to a constant heat and mass flux. The suction velocity and permeability of the porous medium are assumed to time dependent and are of the form as shown in the figure:

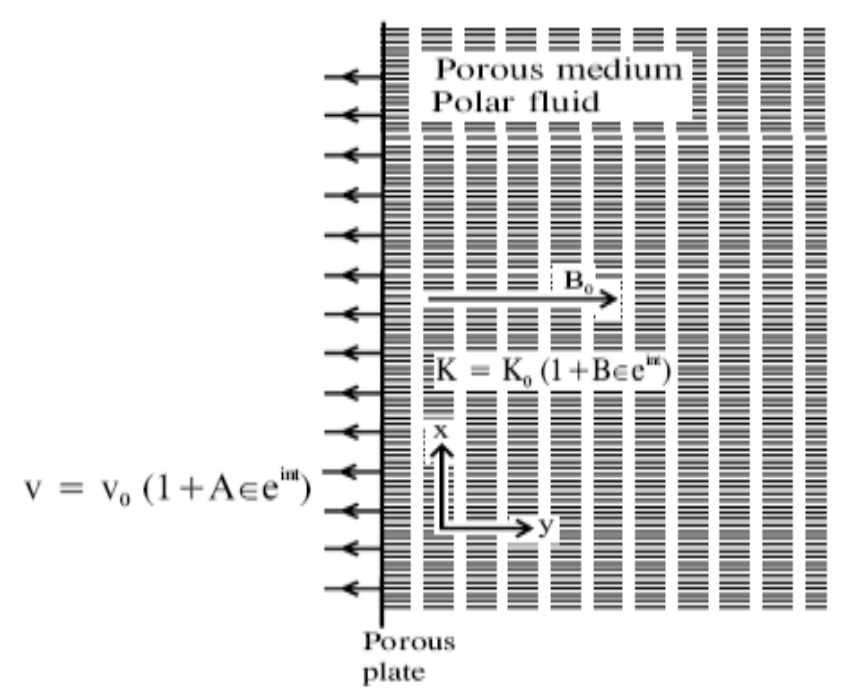

Figure: Schematic diagram.

where $\mathrm{K}_{0}$ is the mean permeability of the medium, $\mathrm{v}_{0}$ is the mean suction velocity, $\mathrm{n}$ is the frequency of fluctuation, $\mathrm{t}$ the time and $\square(<<1), \mathrm{A}$ and $\mathrm{B}$ are constants.

The axis of $\mathrm{x}$ is taken along the plate in vertically upward direction and $\mathrm{y}$-axis is taken normal to the plate. Under these conditions and using the Boussineque's approximation, governing equations of the flow are given by:

$$
\begin{aligned}
& \frac{\partial \mathrm{u}}{\partial \mathrm{t}}-\mathrm{v}_{0}\left(1+\mathrm{A} \in \mathrm{e}^{\mathrm{int}}\right) \frac{\partial \mathrm{u}}{\partial \mathrm{y}}=\mathrm{g} \beta\left(\mathrm{T}-\mathrm{T}_{\infty}\right)+\mathrm{g} \beta^{*}\left(\mathrm{C}-\mathrm{C}_{\infty}\right)+\left(\mathrm{v}+\mathrm{v}_{\mathrm{r}}\right) \frac{\partial^{2} \mathrm{u}}{\partial \mathrm{y}^{2}}+ \\
& \quad 2 \mathrm{v}_{\mathrm{r}} \frac{\partial \omega}{\partial \mathrm{y}}-\frac{\mathrm{v}}{\mathrm{K}_{0}\left(1+\mathrm{B} \in \mathrm{e}^{\mathrm{int}}\right)} \mathrm{u}-\frac{\sigma \mathrm{B}_{0}^{2}}{\rho} \mathrm{u} \\
& \frac{\partial \omega}{\partial \mathrm{t}}-\mathrm{v}_{0}\left(1+\mathrm{A} \in \mathrm{e}^{\mathrm{int}}\right) \frac{\partial \omega}{\partial \mathrm{y}}=\frac{\gamma}{\mathrm{I}} \frac{\partial^{2} \omega}{\partial \mathrm{y}^{2}} \\
& \frac{\partial \mathrm{T}}{\partial \mathrm{t}}-\mathrm{v}_{0}\left(1+\mathrm{A} \in \mathrm{e}^{\mathrm{int}}\right) \frac{\partial \mathrm{T}}{\partial \mathrm{y}}=\frac{\kappa}{\rho \mathrm{C}_{\mathrm{P}}} \frac{\partial^{2} \mathrm{~T}}{\partial \mathrm{y}^{2}}-\frac{1}{\rho \mathrm{C}_{\mathrm{P}}} \frac{\partial \mathrm{q}_{\mathrm{r}}}{\partial \mathrm{y}}
\end{aligned}
$$




$$
\frac{\partial \mathrm{C}}{\partial \mathrm{t}}-\mathrm{v}_{0}\left(1+\mathrm{A} \in \mathrm{e}^{\mathrm{int}}\right) \frac{\partial \mathrm{C}}{\partial \mathrm{y}}=\mathrm{D} \frac{\partial^{2} \mathrm{C}}{\partial \mathrm{y}^{2}}
$$

where $\mathrm{u}, \omega, \mathrm{T}$ and $\mathrm{C}$ are velocity, angular velocity, temperature and species concentration respectively of the fluid particles, $\mathrm{g}$ is acceleration due to gravity, $\square$ is coefficient of volume expansion, $\square$ is coefficient of species concentration expansion, $\square, \square, \mathrm{v}_{\mathrm{r}}, \square \mathrm{C}_{\mathrm{P}}, \square, \mathrm{B}, \mathrm{D}, \mathrm{K}$ are density, kinematic viscosity, rotational kinematic viscosity, thermal conductivity, specific heat at constant pressure, electrical conductivity, magnetic field, mass diffusivity and permeability of porous medium respectively. I a scalar constant equal to moment of Inertia of unit mass and

$$
\gamma=\mathrm{C}_{\mathrm{a}}+\mathrm{C}_{\mathrm{d}}
$$

where $C_{a}$ and $C_{d}$ are coefficient of couple stress viscosities.

The local radiant heat for the case of an optically thin grey gas in expressed by England and Emery [21] as

$$
\frac{\partial \mathrm{q}_{\mathrm{r}}}{\partial \mathrm{y}}=-4 \mathrm{a}^{*} \sigma^{*}\left(\mathrm{~T}_{\infty}^{4}-\mathrm{T}^{4}\right)
$$

where $\mathrm{T}^{4} \cong 4 \mathrm{~T}_{\infty}^{3} \mathrm{~T}-3 \mathrm{~T}_{\infty}^{4}$

* is Stefan-Boltzmann constant and a* is absorption coefficient.

The boundary conditions are:

$$
\left.\begin{array}{l}
\mathrm{y}=0: u=L_{1} \frac{\partial u}{\partial y}, \frac{\partial \omega}{\partial y}=-\frac{\partial^{2} \mathrm{u}}{\partial \mathrm{y}^{2}}, \frac{\partial \mathrm{T}}{\partial \mathrm{y}}=-\frac{\mathrm{q}}{\kappa}, \frac{\partial \mathrm{C}}{\partial \mathrm{y}}=-\frac{\mathrm{m}}{\mathrm{D}} \\
\mathrm{y} \rightarrow \infty: \mathrm{u} \rightarrow 0, \quad \omega \rightarrow 0, \quad \mathrm{~T} \rightarrow \mathrm{T}_{\infty}, \quad \mathrm{C} \rightarrow \mathrm{C}_{\infty}
\end{array}\right\}
$$

where $\mathrm{L}_{1}=\left(2-\mathrm{m}_{1} / \mathrm{m}_{1}\right) \mathrm{L}, \mathrm{m}_{1}$ being the Maxwell's reflection coefficient and $\mathrm{L}$ the free path.

On introducing the following non-dimensional quantities:

$\mathrm{y}^{*}=\frac{\mathrm{yv}_{0}}{\mathrm{v}}, \quad \mathrm{t}^{*}=\frac{\mathrm{tv}_{0}^{2}}{4 \mathrm{v}}, \quad \mathrm{n}^{*}=\frac{4 \mathrm{un}}{\mathrm{v}_{0}^{2}}$

$\mathrm{u}^{*}=\frac{\mathrm{u}}{\mathrm{v}_{0}}, \quad \omega^{*}=\frac{\mathrm{v \omega}}{\mathrm{v}_{0}^{2}}$,

$\theta=\frac{\left(\mathrm{T}-\mathrm{T}_{\infty}\right) \kappa \mathrm{v}_{0}}{u \mathrm{q}}, \mathrm{C}^{*}=\frac{\left(\mathrm{C}-\mathrm{C}_{\infty}\right) \mathrm{Dv}_{0}}{\mathrm{um}}$,

$\mathrm{K}^{*}=\frac{\mathrm{K}_{0} \mathrm{v}_{0}^{2}}{\mathrm{v}^{2}}$ (permeability parameter),

equations (1) to (4) in non-dimensional form, after dropping the asterisks over them, reduces to

$$
\begin{aligned}
& \frac{1}{4} \frac{\partial \mathrm{u}}{\partial \mathrm{t}}-\left(1+\mathrm{A} \in \mathrm{e}^{\mathrm{int}}\right) \frac{\partial \mathrm{u}}{\partial \mathrm{y}}= \mathrm{Gr} \theta+\mathrm{GcC}+\left(1+\alpha_{1}\right) \frac{\partial^{2} \mathrm{u}}{\partial \mathrm{y}^{2}}+ \\
&-\left\{\mathrm{M}+\frac{1}{\mathrm{~K}\left(1+\mathrm{B} \in \mathrm{e}^{\mathrm{int}}\right)}\right\} \mathrm{u} \\
& \frac{1}{4} \frac{\partial \omega}{\partial \mathrm{t}}-\left(1+\mathrm{A} \in \mathrm{e}^{\mathrm{int}}\right) \frac{\partial \omega}{\partial \mathrm{y}}=\frac{1}{\beta_{1}} \frac{\partial^{2} \omega}{\partial \mathrm{y}^{2}} \\
& \frac{1}{4} \frac{\partial \theta}{\partial \mathrm{t}}-\left(1+\mathrm{A} \in \mathrm{e}^{\mathrm{int}}\right) \frac{\partial \theta}{\partial \mathrm{y}}=\frac{1}{\operatorname{Pr}} \frac{\partial^{2} \theta}{\partial \mathrm{y}^{2}}-\frac{\mathrm{R}}{\operatorname{Pr}} \theta \\
& \frac{1}{4} \frac{\partial \mathrm{C}}{\partial \mathrm{t}}-\left(1+\mathrm{A} \in \mathrm{e}^{\mathrm{int}}\right) \frac{\partial \mathrm{C}}{\partial \mathrm{y}}=\frac{1}{\mathrm{Sc}} \frac{\partial^{2} \mathrm{C}}{\partial \mathrm{y}^{2}}
\end{aligned}
$$


where

$\mathrm{h}_{1}=\frac{\mathrm{v}_{0} \mathrm{~L}_{1}}{\mathrm{v}}$ (velocity slip parameter $)$,

$\mathrm{Gr}=\frac{\mathrm{g} \beta \mathrm{v}^{2} \mathrm{q}}{\kappa \mathrm{v}_{0}^{4}}($ thermal Grashof number $)$,

$\mathrm{Gc}=\frac{\mathrm{g} \beta * \mathrm{v}^{2} \mathrm{~m}}{\mathrm{Dv_{0 } ^ { 4 }}}$ (mass Grashof number),

$M=\frac{\sigma B_{0}^{2} v}{\rho v_{0}^{2}} \quad$ (magnetic parameter),

$\alpha_{1}=\frac{U_{r}}{v} \quad$ (rotational parameter),

$\beta_{1}=\frac{\mathrm{Iv}}{\gamma} \quad$ (couple stress parameter),

$\operatorname{Pr}=\frac{\mu C_{P}}{\kappa} \quad$ (Prandtl number),

$\mathrm{Sc}=\frac{\mathrm{U}}{\mathrm{D}} \quad$ (Schmidt number),

$\mathrm{R}=\frac{16 \mathrm{a}^{*} \sigma^{*} \mathrm{v}^{2} \mathrm{~T}_{\infty}^{3}}{\mathrm{v}_{\mathrm{o}}^{2} \kappa}$ (radiation parameter).

With corresponding boundary conditions as:

$$
\left.\begin{array}{llll}
y=0 \quad: u=h_{1} \frac{\partial u}{\partial y}, & \frac{\partial \omega}{\partial y}=-\frac{\partial^{2} u}{\partial y^{2}}, & \frac{\partial \theta}{\partial y}=-1, & \frac{\partial C}{\partial y}=-1 \\
y \rightarrow \infty: \quad u \rightarrow 0, & \omega \rightarrow 0, & \theta \rightarrow 0, & C \rightarrow 0
\end{array}\right\}
$$

\section{Solution of the problem}

Since $\in(<<1)$ is very small and to reduce the system of partial differential equations (6) to (9) into ordinary differential equations, we assume:

$$
\mathrm{f}(\mathrm{y}, \mathrm{t})=\mathrm{f}_{0}(\mathrm{y})+\in \mathrm{e}^{\mathrm{int}} \mathrm{f}_{1}(\mathrm{y})
$$

Where f stands for $\mathrm{u}, \square, \square$ and $\mathrm{C}$.

Substituting expression (11) in (6) to (9) and comparing the coefficients of identical powers of $\in$ we obtain

$$
\begin{aligned}
& \mathrm{u}_{0}^{\prime \prime}+\frac{1}{\left(1+\alpha_{1}\right)} \mathrm{u}_{0}^{\prime}-\frac{\left(\mathrm{M}+\frac{1}{\mathrm{~K}}\right)}{\left(1+\alpha_{1}\right)} \mathrm{u}_{0}=-\frac{\mathrm{Gr}}{\left(1+\alpha_{1}\right)} \theta_{0}-\frac{\mathrm{Gc}}{\left(1+\alpha_{1}\right)} \mathrm{C}_{0} \\
& -\frac{2 \alpha_{1}}{\left(1+\alpha_{1}\right)} \omega_{0}^{\prime} \\
& \mathrm{u}_{1}^{\prime \prime}+\frac{1}{\left(1+\alpha_{1}\right)} \mathrm{u}_{1}^{\prime}-\frac{\left(\mathrm{M}+\frac{1}{\mathrm{~K}}+\frac{\mathrm{in}}{4}\right)}{\left(1+\alpha_{1}\right)} \mathrm{u}_{1}=-\frac{\mathrm{Gr}}{\left(1+\alpha_{1}\right)} \theta_{1}-\frac{\mathrm{Gc}}{\left(1+\alpha_{1}\right)} \mathrm{C}_{1} \\
& \omega_{0}^{\prime \prime}+\beta_{1} \omega_{0}^{\prime}=0 \\
& \omega_{0}^{\prime \prime}+\beta_{1} \omega_{1}^{\prime}-\frac{\mathrm{in} \beta_{1}}{4} \omega_{1}=-\mathrm{A} \beta_{1} \omega_{0}^{\prime} \\
& \theta_{0}^{\prime \prime}+\operatorname{Pr} \theta_{0}^{\prime}-\mathrm{R} \theta_{0}=0
\end{aligned}
$$


$\theta_{1}^{\prime \prime}+\operatorname{Pr} \theta_{1}^{\prime}-\left(\mathrm{R}+\frac{\operatorname{Prin}}{4}\right) \theta_{1}=-\mathrm{A} \operatorname{Pr} \theta_{0}^{\prime}$

$\mathrm{C}_{0}^{\prime \prime}+\mathrm{Sc} \mathrm{C}_{0}^{\prime}=0$

$\mathrm{C}_{1}^{\prime \prime}+\mathrm{Sc} \mathrm{C}_{1}^{\prime}-\frac{\mathrm{Sc} \text { in }}{4} \mathrm{C}_{1}=-\mathrm{AScC}_{0}^{\prime}$

with boundary conditions:

$$
\left.\begin{array}{rl}
y=0: u_{0}=h_{1} u_{0}^{\prime}, \omega_{0}^{\prime}=-u_{0}^{\prime \prime}, & \theta_{0}^{\prime}=-1, C_{0}^{\prime}=-1 \\
u_{1}=h_{1} u_{1}^{\prime}, & \omega_{1}^{\prime}=-u_{1}^{\prime \prime}, \quad \theta_{1}^{\prime}=0, \quad C_{1}^{\prime}=0 \\
y \rightarrow \infty: u_{0} \rightarrow 0, \quad \omega_{0} \rightarrow 0, \quad \theta_{0} \rightarrow 0, \quad C_{0} \rightarrow 0 \\
u_{1} \rightarrow 0, \quad \omega_{1} \rightarrow 0, \quad \theta_{1} \rightarrow 0, \quad C_{1} \rightarrow 0
\end{array}\right\}
$$

Solving (12) to (19) under boundary conditions (20) we get:

$$
\begin{aligned}
\mathrm{u}_{0}= & \mathrm{m}_{7} \mathrm{e}^{\mathrm{x}_{9} \mathrm{y}}+\mathrm{J}_{3} \mathrm{e}^{\mathrm{x}_{3} \mathrm{y}}+\mathrm{J}_{\mathrm{u}} \overline{\mathrm{e}}^{\mathrm{Scy}}+\mathrm{J}_{5} \overline{\mathrm{e}}^{\beta_{1} \mathrm{y}} \\
\mathrm{u}_{1}= & \mathrm{m}_{8} \mathrm{e}^{\mathrm{x}_{11} \mathrm{y}}+\mathrm{J}_{7} \mathrm{e}^{\mathrm{x}_{5} \mathrm{y}}+\mathrm{J}_{8} \mathrm{e}^{\mathrm{x}_{7} \mathrm{y}}+\mathrm{J}_{9} \mathrm{e}^{\mathrm{x}_{1} \mathrm{y}}+\mathrm{J}_{10} \mathrm{e}^{\mathrm{x}_{3} \mathrm{y}}+\mathrm{J}_{11} \overline{\mathrm{e}}^{\text {Scy }} \\
& +\mathrm{J}_{12} \overline{\mathrm{e}}^{\beta_{1} \mathrm{y}}+\mathrm{J}_{13} \mathrm{e}^{\mathrm{x}_{9} \mathrm{y}} \\
\omega_{0}= & \mathrm{m}_{1} \overline{\mathrm{e}}^{\beta_{1} \mathrm{y}} \\
\omega_{1}= & \mathrm{m}_{2} \mathrm{e}^{\mathrm{x}_{1} \mathrm{y}}+\mathrm{J}_{6} \overline{\mathrm{e}}^{\beta_{1} \mathrm{y}} \\
\theta_{0}= & \mathrm{m}_{3} \mathrm{e}^{\mathrm{x}_{3} \mathrm{y}} \\
\theta_{1}= & \mathrm{m}_{4} \mathrm{e}^{\mathrm{x}_{5} \mathrm{y}}+\mathrm{J}_{1} \mathrm{e}^{\mathrm{x}_{3} \mathrm{y}} \\
\mathrm{C}_{0}= & \mathrm{m}_{5} \overline{\mathrm{e}}^{\mathrm{Scy}} \\
\mathrm{C}_{1}= & \mathrm{m}_{6} \mathrm{e}^{\mathrm{x}_{7} \mathrm{y}}+\mathrm{J}_{2} \overline{\mathrm{e}}^{\mathrm{Scy}}
\end{aligned}
$$

Now substituting equations (21) to (28) in (11) and separating the real and imaginary parts, we get the expressions of real part for velocity, angular velocity, temperature and concentration as:

$$
\begin{aligned}
& \mathrm{u}=\mathrm{u}_{0}(\mathrm{y})+\in\left(\mathrm{Mr}_{1} \cos \mathrm{t}-\mathrm{Mi}_{1} \sin \mathrm{nt}\right) \\
& \omega=\omega_{0}(\mathrm{y})+\in\left(\mathrm{Mr}_{2} \cos \mathrm{nt}-\mathrm{Mi}_{2} \sin \mathrm{nt}\right) \\
& \theta=\theta_{0}(\mathrm{y})+\in\left(\mathrm{Mr}_{3} \cos \mathrm{nt}-\mathrm{Mi}_{3} \sin \mathrm{nt}\right) \\
& \mathrm{C}=\mathrm{C}_{0}(\mathrm{y})+\in\left(\mathrm{Mr}_{4} \cos \mathrm{nt}-\mathrm{Mi}_{4} \sin \mathrm{nt}\right)
\end{aligned}
$$

where

$$
\begin{aligned}
& \mathrm{Mr}_{1}=\mathrm{d}_{66}+\mathrm{d}_{16}+\mathrm{d}_{18}+\mathrm{d}_{20}+\mathrm{d}_{22}+\mathrm{d}_{24}+\mathrm{d}_{26} \\
& \mathrm{Mi}_{1}=\mathrm{d}_{67}+\mathrm{d}_{17}+\mathrm{d}_{19}+\mathrm{d}_{21}+\mathrm{d}_{23}+\mathrm{d}_{25}+\mathrm{d}_{27}+\mathrm{d}_{28} \\
& \mathrm{Mr}_{2}=\mathrm{e}^{\mathrm{d}_{8} \mathrm{y} / 2}\left(\mathrm{~d}_{6} \mathrm{C}_{1}-\mathrm{d}_{7} \mathrm{C}_{2}\right) \\
& \mathrm{Mi}_{2}=\mathrm{e}^{\mathrm{d}_{8} \mathrm{y} / 2}\left(\mathrm{~d}_{6} \mathrm{C}_{2}+\mathrm{d}_{7} \mathrm{C}_{1}\right)+\frac{4 \mathrm{Au}_{0}^{\prime \prime}(0)}{\mathrm{n}} \mathrm{e}^{-\beta_{1} \mathrm{y}} \\
& \mathrm{Mr}_{3}=\mathrm{d}_{34}+\mathrm{d}_{36} \quad, \quad \mathrm{Mi}_{3}=\mathrm{d}_{35}+\mathrm{d}_{37} \\
& \mathrm{Mr}_{4}=\mathrm{d}_{42}, \quad \mathrm{Mi}_{4}=\mathrm{d}_{43}+\mathrm{d}_{44}
\end{aligned}
$$

When velocity component $u$ is obtained, now we can now obtain the important parameter viz. skin friction in real part as: 


$$
\begin{gathered}
\mathrm{C}_{\mathrm{f}}=\frac{\tau_{\mathrm{w}}}{\rho \mathrm{v}_{0}^{2}}=\left(1+\alpha_{1}\right)\left\{\left(\mathrm{m}_{7} \mathrm{x}_{9}+\mathrm{J}_{3} \mathrm{x}_{3}-\mathrm{J}_{4} \mathrm{Sc}-\mathrm{J}_{5} \beta_{1}\right)+\right. \\
\left.\in\left(\mathrm{t}_{16} \cos \mathrm{nt}-\mathrm{t}_{17} \sin \mathrm{nt}\right)\right\}
\end{gathered}
$$

\section{Nusselt Number}

Another important physical parameter of interest viz. Nusselt number in dimensionless form is:

$$
\mathrm{Nu}=\frac{1}{\theta(0)}
$$

where

$\theta(0)=\mathrm{m}_{3}+\in\left\{\left(\mathrm{t}_{18} \cos \mathrm{nt}-\mathrm{t}_{19} \sin \mathrm{nt}\right)+\mathrm{i}\left(\mathrm{t}_{18} \sin \mathrm{nt}+\mathrm{t}_{19} \cos \mathrm{nt}\right)\right\}$.

Taking the real part only, we get:

$$
\mathrm{Nu}=\frac{\mathrm{t}_{20}}{\mathrm{t}_{20}^{2}+\mathrm{t}_{21}^{2}} \text {. }
$$

Where

$$
\begin{aligned}
& \mathrm{x}_{1}=\frac{-\beta_{1}-\sqrt{\beta_{1}^{2}+\mathrm{in} \beta_{1}}}{2}, \quad \mathrm{x}_{2}=\frac{-\beta_{1}+\sqrt{\beta_{1}^{2}+\mathrm{in} \beta_{1}}}{2} \\
& \mathrm{x}_{3}=\frac{-\operatorname{Pr}-\sqrt{\operatorname{Pr}^{2}+4 \mathrm{R}}}{2}, \quad \mathrm{x}_{4}=\frac{-\operatorname{Pr}+\sqrt{\operatorname{Pr}^{2}+4 \mathrm{R}}}{2} \\
& \mathrm{x}_{5}=\frac{-\operatorname{Pr}-\sqrt{\operatorname{Pr}^{2}+4\left(\mathrm{R}+\frac{\operatorname{Prin}}{4}\right)}}{2}, \quad \mathrm{x}_{6}=\frac{-\operatorname{Pr}+\sqrt{\operatorname{Pr}^{2}+4\left(\mathrm{R}+\frac{\operatorname{Prin}}{4}\right)}}{2} \\
& \mathrm{x}_{7}=\frac{-\mathrm{Sc}-\sqrt{\mathrm{Sc}^{2}+\mathrm{Scin}}}{2}, \mathrm{x}_{8}=\frac{-\mathrm{Sc}+\sqrt{\mathrm{Sc}^{2}+\mathrm{Scin}}}{2} \\
& \mathrm{x}_{9}=\frac{-1-\sqrt{1+4\left(\mathrm{M}+\frac{1}{\mathrm{~K}}\right)\left(1+\alpha_{1}\right)}}{2\left(1+\alpha_{1}\right)}, \quad \mathrm{x}_{10}=\frac{-1+\sqrt{1+4\left(\mathrm{M}+\frac{1}{\mathrm{~K}}\right)\left(1+\alpha_{1}\right)}}{2\left(1+\alpha_{1}\right)} \\
& \mathrm{x}_{11}=\frac{-1-\sqrt{1+4\left(\mathrm{M}+\frac{1}{\mathrm{~K}}+\frac{\mathrm{in}}{4}\right)\left(1+\alpha_{1}\right)}}{2\left(1+\alpha_{1}\right)} \quad, \quad \mathrm{x}_{12}=\frac{-1+\sqrt{1+4\left(\mathrm{M}+\frac{1}{\mathrm{~K}}+\frac{\mathrm{in}}{4}\right)\left(1+\alpha_{1}\right)}}{2\left(1+\alpha_{1}\right)} \\
& \mathrm{m}_{1}=\frac{\mathrm{u}_{0}^{\prime \prime}(0)}{\beta_{1}}, \quad \mathrm{~m}_{2}=\frac{1}{\mathrm{x}_{1}}\left[-\mathrm{u}_{1}^{\prime \prime}(0)+\mathrm{J}_{6} \beta_{1}\right] \\
& \mathrm{m}_{3}=-\frac{1}{\mathrm{x}_{3}} \quad, \quad \mathrm{~m}_{4}=\frac{-\mathrm{Ax}_{3}}{\mathrm{x}_{5}\left(\mathrm{x}_{3}-\mathrm{x}_{6}\right)\left(\mathrm{x}_{3}-\mathrm{x}_{5}\right)} \\
& \mathrm{m}_{5}=\frac{1}{\mathrm{Sc}} \quad, \quad \mathrm{m}_{6}=\frac{\mathrm{A} \mathrm{Sc}}{\mathrm{x}_{7}\left(\mathrm{Sc}+\mathrm{x}_{7}\right)\left(\mathrm{Sc}+\mathrm{x}_{8}\right)} \\
& \mathrm{m}_{7}=\frac{\mathrm{J}_{3}\left(\mathrm{~h}_{1} \mathrm{x}_{3-1}\right)-\mathrm{J}_{4}\left(1+\mathrm{h}_{1} \mathrm{Sc}\right)-\mathrm{J}_{5}\left(1+\mathrm{h}_{1} \beta_{1}\right)}{\left(1-\mathrm{h}_{1} \mathrm{x}_{9}\right)}
\end{aligned}
$$




$$
\begin{aligned}
& \mathbf{J}_{3}=\frac{-\mathrm{Gr} \mathrm{m}_{3}}{\left(1+\alpha_{1}\right)\left(\mathrm{x}_{3}-\mathrm{x}_{9}\right)\left(\mathrm{x}_{3}-\mathrm{x}_{10}\right)}, \quad \mathrm{J}_{4}=\frac{-\mathrm{Gc} \mathrm{m}_{5}}{\left(1+\alpha_{1}\right)\left(\mathrm{Sc}+\mathrm{x}_{10}\right)\left(\mathrm{Sc}+\mathrm{x}_{9}\right)} \\
& \mathrm{J}_{5}=\frac{2 \alpha_{1} \mathrm{~m}_{1} \beta_{1}}{\left(1+\alpha_{1}\right)\left(\beta_{1}+\mathrm{x}_{10}\right)\left(\beta_{1}+\mathrm{x}_{9}\right)}, \quad \mathrm{J}_{1}=\frac{\mathrm{APr}}{\left(\mathrm{x}_{3}-\mathrm{x}_{5}\right)\left(\mathrm{x}_{3}-\mathrm{x}_{6}\right)} \\
& \mathrm{J}_{2}=\frac{\mathrm{ASc}}{\left(\mathrm{Sc}+\mathrm{x}_{7}\right)\left(\mathrm{Sc}+\mathrm{x}_{8}\right)}, \quad \mathrm{J}_{6}=\frac{\mathrm{Am}_{1} \beta_{1}^{2}}{\left(\beta_{1}+\mathrm{x}_{2}\right)\left(\beta_{1}+\mathrm{x}_{1}\right)} \\
& \mathrm{J}_{7}=\frac{-\mathrm{Gr} \mathrm{m}_{4}}{\left(1+\alpha_{1}\right)\left(\mathrm{x}_{5}-\mathrm{x}_{12}\right)\left(\mathrm{x}_{5}-\mathrm{x}_{11}\right)} \quad, \quad \mathrm{J}_{8}=\frac{-\mathrm{Gc} \mathrm{m}_{6}}{\left(1+\alpha_{1}\right)\left(\mathrm{x}_{7}-\mathrm{x}_{12}\right)\left(\mathrm{x}_{7}-\mathrm{x}_{11}\right)} \\
& \mathrm{J}_{9}=\frac{-2 \alpha_{1} \mathrm{~m}_{2} \mathrm{x}_{1}}{\left(1+\alpha_{1}\right)\left(\mathrm{x}_{1}-\mathrm{x}_{11}\right)\left(\mathrm{x}_{1}-\mathrm{x}_{12}\right)}, \quad \mathrm{J}_{10}=\frac{\mathrm{b}_{1}}{\left(1+\alpha_{1}\right)\left(\mathrm{x}_{3}-\mathrm{x}_{11}\right)\left(\mathrm{x}_{3}-\mathrm{x}_{12}\right)} \\
& \mathrm{J}_{11}=\frac{\mathrm{b}_{2}}{\left(1+\alpha_{1}\right)\left(\mathrm{Sc}+\mathrm{x}_{11}\right)\left(\mathrm{Sc}+\mathrm{x}_{12}\right)}, \mathrm{J}_{12}=\frac{\mathrm{b}_{3}}{\left(1+\alpha_{1}\right)\left(\beta_{1}+\mathrm{x}_{11}\right)\left(\beta_{1}+\mathrm{x}_{12}\right)} \\
& \mathrm{J}_{13}=\frac{\mathrm{b}_{4} \mathrm{~m}_{7}}{\left(1+\alpha_{1}\right)\left(\mathrm{x}_{9}-\mathrm{x}_{11}\right)\left(\mathrm{x}_{9}-\mathrm{x}_{12}\right)}, \quad \mathrm{b}_{1}=\left(-\mathrm{Gr} \mathrm{J}_{1}-\mathrm{AJ}_{3} \mathrm{x}_{3}-\frac{\mathrm{J}_{3}}{\mathrm{~K}}\right) \\
& \mathrm{b}_{2}=\left(-\mathrm{Gc} \mathrm{J}_{2}+\mathrm{AJ}_{4} \mathrm{Sc}-\frac{\mathrm{J}_{4}}{\mathrm{~K}}\right), \mathrm{b}_{3}=2 \alpha_{1} \beta_{1} \mathrm{~J}_{6}+\mathrm{AJ}_{5} \beta_{1}-\frac{\mathrm{J}_{5}}{\mathrm{~K}} \\
& \mathrm{~b}_{4}=-\mathrm{Ax}_{9}-\frac{\mathrm{B}}{\mathrm{K}} \\
& A_{1}=\left(\frac{\operatorname{Pr} n}{4}\right)\left(\frac{\left(\operatorname{Pr}\left(1+\alpha_{1}\right)-1\right)}{2} \sqrt{\frac{\sqrt{\left(\operatorname{Pr}^{2}+4 R\right)^{2}+\operatorname{Pr}^{2} n^{2}}-\left(\operatorname{Pr}^{2}+4 R\right)}{2}}+\frac{\left(\operatorname{Pr}\left(1+\alpha_{1}\right)-1\right) n}{4}\right)
\end{aligned}
$$$$
\mathrm{B}_{1}=\left(-\frac{\operatorname{Pr} \mathrm{n}}{4}\right)\left(\left(1+\alpha_{1}\right) \frac{\operatorname{Pr}^{2}}{2}+\left(1+\alpha_{1}\right) \mathrm{R}-\frac{\operatorname{Pr}}{2}-\mathrm{M}-\frac{1}{\mathrm{~K}}+\frac{\left(\operatorname{Pr}\left(1+\alpha_{1}\right)-1\right)}{2}\right.
$$$$
\left.\sqrt{\frac{\sqrt{\left(\operatorname{Pr}^{2}+4 R\right)^{2}+\operatorname{Pr}^{2} n^{2}}+\left(\operatorname{Pr}^{2}+4 R\right)}{2}}\right)
$$

$$
\begin{aligned}
& \mathrm{A}_{2}=\left(\frac{\mathrm{n}}{4}\right)\left(\frac{\left(\mathrm{Sc}\left(1+\alpha_{1}\right)-1\right)}{2} \sqrt{\frac{\mathrm{Sc} \sqrt{\mathrm{Sc}^{2}+\mathrm{n}^{2}}-\mathrm{Sc}^{2}}{2}}+\frac{\left(\mathrm{Sc}\left(1+\alpha_{1}\right)-1\right) \mathrm{n}}{4}\right) \\
& \mathrm{B}_{2}=\left(-\frac{\mathrm{n}}{4}\right)\left(\left(1+\alpha_{1}\right) \frac{\mathrm{Sc}^{2}}{2}-\frac{\mathrm{Sc}}{2}-\mathrm{M}-\frac{1}{\mathrm{~K}}+\frac{\left(\mathrm{Sc}\left(1+\alpha_{1}\right)-1\right)}{2}\right) \sqrt{\frac{\mathrm{Sc} \sqrt{\mathrm{Sc}^{2}+\mathrm{n}^{2}}+\mathrm{Sc}^{2}}{2}} \\
& \mathrm{~A}_{3}=\frac{\operatorname{Pr}^{2}}{4}-\frac{1}{4} \sqrt{\frac{\sqrt{\left(\mathrm{Pr}^{2}+4 \mathrm{R}\right)^{2}+\mathrm{Pr}^{2} \mathrm{n}^{2}}+\left(\mathrm{Pr}^{2}+4 \mathrm{R}\right)}{2}}+\mathrm{R}
\end{aligned}
$$




$$
\begin{aligned}
& \mathrm{B}_{3}=\frac{1}{4} \sqrt{\frac{\sqrt{\left(\operatorname{Pr}^{2}+4 R\right)^{2}+\operatorname{Pr}^{2} \mathrm{n}^{2}}-\left(\operatorname{Pr}^{2}+4 R\right)}{2}} \\
& \mathrm{~A}_{4}=\left[\frac{-\mathrm{Gr} \mathrm{A} \mathrm{A}_{3}}{\mathrm{~A}_{3}^{2}+\mathrm{B}_{3}^{2}}-\mathrm{AJ}_{3} \mathrm{x}_{3}-\frac{\mathbf{J}_{3}}{\mathrm{~K}}\right] \\
& \mathrm{B}_{4}=\left(\frac{\mathrm{Gr} \mathrm{A} \mathrm{B}_{3}}{\mathrm{~A}_{3}^{2}+\mathrm{B}_{3}^{2}}\right) \\
& \mathrm{A}_{5}=\left(1+\alpha_{1}\right) \frac{\operatorname{Pr}^{2}}{2}-\frac{\operatorname{Pr}}{2}+\mathrm{R}\left(1+\alpha_{1}\right)-\mathrm{M}-\frac{1}{\mathrm{~K}}+\left(\operatorname{Pr}\left(1+\alpha_{1}\right)-1\right) \frac{\sqrt{\operatorname{Pr}^{2}+4 \mathrm{R}}}{2} \\
& \mathrm{~B}_{5}=\frac{\mathrm{n}}{4}
\end{aligned}
$$$$
\mathrm{A}_{6}=\mathrm{AJ}_{4} \mathrm{Sc}-\frac{\mathrm{J}_{4}}{\mathrm{~K}}, \mathrm{~B}_{6}=\frac{4 \mathrm{Gc} \mathrm{A}}{\mathrm{Sc} \mathrm{n}}
$$$$
\mathrm{A}_{7}=\left(1+\alpha_{1}\right) \mathrm{Sc}^{2}-\mathrm{Sc}-\mathrm{M}-\frac{1}{\mathrm{~K}}
$$$$
\mathrm{A}_{8}=\mathrm{A} \mathrm{J}_{5} \beta_{1}-\frac{\mathrm{J}_{5}}{\mathrm{~K}} \quad, \quad \mathrm{~B}_{7}=\frac{8 \alpha_{1} \mathrm{~A} \beta_{1} \mathrm{u}_{0}^{\prime \prime}(\mathrm{o})}{\mathrm{n}}
$$$$
\mathrm{A}_{9}=\left(1+\alpha_{1}\right) \beta_{1}^{2}-\beta_{1}-\mathrm{M}-\frac{1}{\mathrm{~K}}, \quad \mathrm{~A}_{10}=\frac{4 \mathrm{~b}_{4} \mathrm{~m}_{7} \mathrm{x}_{9}^{2}}{\mathrm{n}}
$$$$
\mathrm{A}_{11}=\alpha_{1} \beta_{1}\left(\beta_{1}+\sqrt{\frac{\beta_{1} \sqrt{\beta_{1}^{2}+\mathrm{n}^{2}}+\beta_{1}^{2}}{2}}\right)
$$$$
\mathrm{B}_{8}=\alpha_{1} \beta_{1}\left(\beta_{1}+\sqrt{\frac{\beta_{1} \sqrt{\beta_{1}^{2}+\mathrm{n}^{2}}-\beta_{1}^{2}}{2}}\right)
$$$$
A_{12}=\left(\left(1+\alpha_{1}\right) \frac{\beta_{1}^{2}}{2}-\frac{\beta_{1}}{2}-M-\frac{1}{K}+\frac{\left(\beta_{1}\left(1+\alpha_{1}\right)-1\right)}{2} \sqrt{\frac{\beta_{1} \sqrt{\beta_{1}^{2}+\mathbf{n}^{2}}+\beta_{1}^{2}}{2}}\right)
$$$$
\mathrm{B}_{9}=\left(\left(\beta_{1}\left(1+\alpha_{1}\right)-1\right) \frac{\mathrm{n}}{4}+\frac{\left(\beta_{1}\left(1+\alpha_{1}\right)-1\right)}{2} \sqrt{\frac{\beta_{1} \sqrt{\beta_{1}^{2}+\mathrm{n}^{2}}-\beta_{1}^{2}}{2}}\right)
$$$$
\mathrm{A}_{13}=-\frac{1}{2}\left(\beta_{1}+\sqrt{\frac{\beta_{1} \sqrt{\beta_{1}^{2}+\mathrm{n}^{2}}+\beta_{1}^{2}}{2}}\right), \quad \mathrm{B}_{10}=-\frac{1}{2} \sqrt{\frac{\beta_{1} \sqrt{\beta_{1}^{2}+\mathrm{n}^{2}}-\beta_{1}^{2}}{2}}
$$

$$
\begin{aligned}
& A_{15}=\left\{\left(\frac{G r A_{3} A_{1}}{A_{1}^{2}+B_{1}^{2}}\right)\left(-\frac{\operatorname{Pr}}{2}-\frac{1}{2} \sqrt{\frac{\sqrt{\left(\operatorname{Pr}^{2}+4 R\right)^{2}+\operatorname{Pr}^{2} n^{2}}+\left(\operatorname{Pr}^{2}+4 R\right)}{2}}\right)\right. \\
& \left.-\left(\frac{\mathrm{Gr} \mathrm{Ax_{3 }} \mathrm{B}_{1}}{\mathrm{~A}_{1}^{2} \mathrm{~B}_{1}^{2}}\right)\left(-\frac{1}{2} \sqrt{\frac{\sqrt{\left(\mathrm{Pr}^{2}+4 \mathrm{R}\right)^{2}+\operatorname{Pr}^{2} \mathrm{n}^{2}}-\left(\operatorname{Pr}^{2}+4 \mathrm{R}\right)}{2}}\right)\right\}
\end{aligned}
$$




$$
\begin{aligned}
& B_{12}=\left(\frac{\mathrm{Gr} \mathrm{Ax}_{3} \mathrm{~A}_{1}}{\mathrm{~A}_{1}^{2}+\mathrm{B}_{1}^{2}}\right)\left(-\frac{\operatorname{Pr}}{2}-\frac{1}{2} \sqrt{\frac{\sqrt{\left(\mathrm{Pr}^{2}+4 \mathrm{R}\right)^{2}+\operatorname{Pr}^{2} \mathrm{n}^{2}}+\left(\mathrm{P}^{2}+4 \mathrm{R}\right)}{2}}\right) \\
& +\left(\frac{\mathrm{Gr} \mathrm{Ax}_{3} \mathrm{~A}_{1}}{\mathrm{~A}_{1}^{2}+\mathrm{B}_{1}^{2}}\right)\left(\frac{1}{2} \sqrt{\frac{\sqrt{\left(\mathrm{Pr}^{2}+4 \mathrm{R}\right)^{2}+\operatorname{Pr}^{2} \mathrm{n}^{2}}-\left(\operatorname{Pr}^{2}+4 \mathrm{R}\right)}{2}}\right) \\
& A_{16}=\left(\frac{-G c A A_{2}}{A_{2}^{2}+B_{2}^{2}}\right)\left(-\frac{S c}{2}-\frac{1}{2} \sqrt{\frac{S c \sqrt{{S c^{2}+n^{2}}^{2} S^{2}}}{2}}\right)+\left(\frac{G c A B_{2}}{A_{2}^{2}+B_{2}^{2}}\right) \\
& \left(\frac{1}{2} \sqrt{\frac{\mathrm{Sc} \sqrt{\mathrm{Sc}^{2}+\mathrm{n}^{2}}-\mathrm{Sc}^{2}}{2}}\right) \\
& B_{13}=\left(\frac{G c A B_{2}}{A_{2}^{2}+B_{2}^{2}}\right)\left(-\frac{S c}{2}-\frac{1}{2} \sqrt{\frac{S c \sqrt{{S c^{2}+n^{2}}^{2} S^{2}}}{2}}\right)+\left(\frac{G c A A_{2}}{A_{2}^{2}+B_{2}^{2}}\right) \\
& \left(\frac{1}{2} \sqrt{\frac{\mathrm{Sc} \sqrt{\mathrm{Sc}^{2}+\mathrm{n}^{2}}-\mathrm{Sc}^{2}}{2}}\right)
\end{aligned}
$$

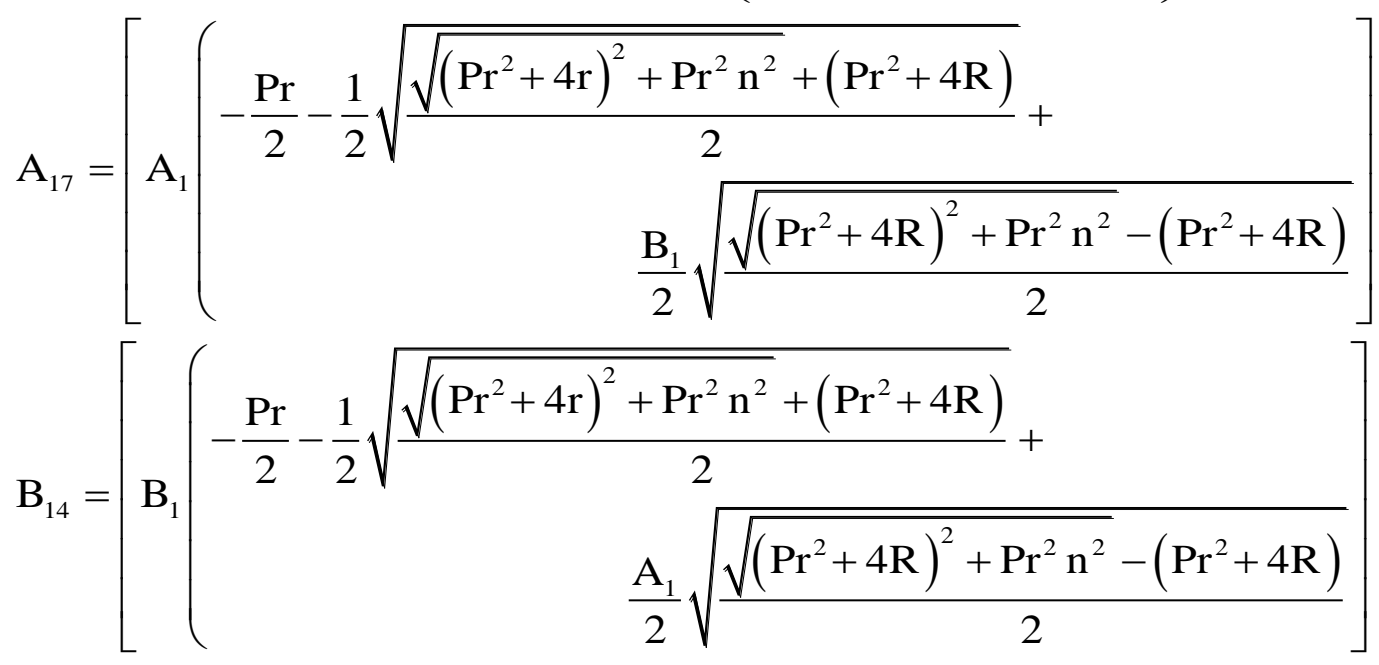

$\mathrm{A}_{18}=\left[\frac{\mathrm{A}_{2}}{2}\left(-\mathrm{Sc}-\sqrt{\frac{\mathrm{Sc} \sqrt{\mathrm{Sc}^{2}+\mathrm{n}^{2}}+\mathrm{Sc}^{2}}{2}}+\frac{\mathrm{B}_{2}}{2} \sqrt{\frac{\mathrm{Sc} \sqrt{\mathrm{Sc}^{2}+\mathrm{n}^{2}}-\mathrm{Sc}^{2}}{2}}\right]\right.$

$\mathrm{B}_{15}=\left[\frac{\mathrm{B}_{2}}{2}\left(-\mathrm{Sc}-\sqrt{\frac{\mathrm{Sc} \sqrt{\mathrm{Sc}^{2}+\mathrm{n}^{2}}+\mathrm{Sc}^{2}}{2}}-\frac{\mathrm{A}_{2}}{2} \sqrt{\frac{\mathrm{Sc}_{\sqrt{\mathrm{Sc}^{2}+\mathrm{n}^{2}}}-\mathrm{Sc}^{2}}{2}}\right]\right.$

$\mathrm{s}_{1}=\frac{\mathrm{GrAx}_{3} \mathrm{~A}_{17}}{\mathrm{~A}_{17}^{2}+\mathrm{B}_{14}^{2}} \quad, \quad \mathrm{~s}_{2}=\frac{\mathrm{GrAx}_{3} \mathrm{~B}_{14}}{\mathrm{~A}_{17}^{2}+\mathrm{B}_{14}^{2}}$

$\mathrm{d}_{46}=\mathrm{s}_{1}\left(+\frac{\mathrm{h}_{1}}{2} \mathrm{~d}_{10}-1\right)+\mathrm{s}_{2} \frac{\mathrm{h}_{1}}{2} \mathrm{~d}_{11}, \quad \mathrm{~d}_{47}=-\mathrm{s}_{2}\left(+\frac{\mathrm{h}_{1}}{2} \mathrm{~d}_{10}-1\right)+\mathrm{s}_{1} \frac{\mathrm{h}_{1}}{2} \mathrm{~d}_{11}$ 


$$
\begin{aligned}
& s_{3}=-\frac{\mathrm{GcAA}_{18}}{\mathrm{~A}_{18}^{2}+\mathrm{B}_{15}^{2}}, \quad \mathrm{~s}_{4}=\frac{\mathrm{GcAB}_{15}}{\mathrm{~A}_{18}^{2}+\mathrm{B}_{15}^{2}} \\
& \mathrm{~d}_{48}=\mathrm{s}_{3}\left(+\frac{\mathrm{h}_{1}}{2} \mathrm{~d}_{12}-1\right)-\mathrm{s}_{4}\left(\frac{\mathrm{h}_{1}}{2} \mathrm{~d}_{13}\right) \\
& \mathrm{d}_{49}=\mathrm{s}_{4}\left(+\frac{\mathrm{h}_{1}}{2} \mathrm{~d}_{12}-1\right)+\mathrm{s}_{3}\left(\frac{\mathrm{h}_{1}}{2} \mathrm{~d}_{13}\right) \text {, } \\
& \mathrm{s}_{7}=\frac{\left(\mathrm{A}_{4} \mathrm{~A}_{5}+\mathrm{B}_{4} \mathrm{~B}_{5}\right)}{\mathrm{A}_{5}^{2}+\mathrm{B}_{5}^{2}} \quad, \quad \mathrm{~s}_{8}=\frac{\left(\mathrm{A}_{4} \mathrm{~B}_{5}-\mathrm{A}_{5} \mathrm{~B}_{4}\right)}{\mathrm{A}_{5}^{2}+\mathrm{B}_{5}^{2}} \\
& \mathrm{~d}_{52}=\mathrm{s}_{7}\left(\mathrm{~h}_{1} \mathrm{x}_{3}-1\right) \quad, \quad \mathrm{d}_{53}=\mathrm{s}_{8}\left(\mathrm{~h}_{1} \mathrm{x}_{3}-1\right) \\
& \mathrm{s}_{9}=\frac{\mathrm{A}_{6} \mathrm{~A}_{7}+\mathrm{B}_{6} \mathrm{~B}_{5}}{\mathrm{~A}_{7}^{2}+\mathrm{B}_{5}^{2}} \quad, \quad \mathrm{~s}_{10}=\frac{\left(\mathrm{A}_{6} \mathrm{~B}_{5}-\mathrm{A}_{7} \mathrm{~B}_{6}\right)}{\mathrm{A}_{7}^{2}+\mathrm{B}_{5}^{2}} \\
& \mathrm{~d}_{54}=-\mathrm{s}_{9}\left(\mathrm{~h}_{1} \mathrm{Sc}+1\right) \quad, \quad \mathrm{d}_{55}=\mathrm{s}_{10}\left(\mathrm{~h}_{1} \mathrm{Sc}+1\right) \\
& \mathrm{s}_{11}=\frac{\left(\mathrm{A}_{8} \mathrm{~A}_{9}-\mathrm{B}_{7} \mathrm{~B}_{5}\right)}{\mathrm{A}_{9}^{2}+\mathrm{B}_{5}^{2}} \quad, \quad \mathrm{~s}_{12}=\frac{\left(\mathrm{A}_{8} \mathrm{~B}_{5}+\mathrm{A}_{9} \mathrm{~B}_{7}\right)}{\mathrm{A}_{9}^{2}+\mathrm{B}_{5}^{2}} \\
& \mathrm{~d}_{56}=-\mathrm{s}_{11}\left(\mathrm{~h}_{1} \beta_{1}+1\right) \quad, \quad \mathrm{d}_{57}=\mathrm{s}_{12}\left(\mathrm{~h}_{1} \beta_{1}+1\right) \\
& \mathrm{d}_{58}=\frac{4 \mathrm{~b}_{4} \mathrm{~m}_{7}}{\mathrm{n}}\left(\mathrm{h}_{1} \mathrm{x}_{9}-1\right) \\
& \mathrm{s}_{15}=\frac{-1}{\left(1+\alpha_{1}\right)}\left(1+\sqrt{\frac{\sqrt{\left(1+4\left(\mathrm{M}+\frac{1}{\mathrm{~K}}\right)\left(1+\alpha_{1}\right)\right)^{2}+\mathrm{n}^{2}\left(1+\alpha_{1}\right)^{2}}+\left(1+4\left(\mathrm{M}+\frac{1}{\mathrm{~K}}\right)\left(1+\alpha_{1}\right)\right)}{2}}\right)
\end{aligned}
$$$$
\mathrm{s}_{16}=\frac{-1}{\left(1+\alpha_{1}\right)}\left(1+\sqrt{\frac{\sqrt{\left(1+4\left(\mathrm{M}+\frac{1}{\mathrm{~K}}\right)\left(1+\alpha_{1}\right)\right)^{2}+\mathrm{n}^{2}\left(1+\alpha_{1}\right)^{2}}-\left(1+4\left(\mathrm{M}+\frac{1}{\mathrm{~K}}\right)\left(1+\alpha_{1}\right)\right)}{2}}\right)
$$$$
\mathrm{d}_{60}=1-\frac{\mathrm{h}_{1} \mathrm{~s}_{15}}{2} \quad, \quad \mathrm{~d}_{61}=\frac{\mathrm{h}_{1} \mathrm{~s}_{16}}{2}
$$$$
\mathrm{d}_{62}=\left(\mathrm{d}_{46}+\mathrm{d}_{48}+\mathrm{d}_{52}+\mathrm{d}_{54}+\mathrm{d}_{56}\right) \quad, \quad \mathrm{d}_{63}=\left(\mathrm{d}_{47}+\mathrm{d}_{49}+\mathrm{d}_{53}-\mathrm{d}_{55}-\mathrm{d}_{57}+\mathrm{d}_{58}\right)
$$$$
\mathrm{d}_{64}=\frac{\left(\mathrm{d}_{62} \mathrm{~d}_{60}+\mathrm{d}_{63} \mathrm{~d}_{61}\right)}{\mathrm{d}_{60}^{2}+\mathrm{d}_{61}^{2}} \quad, \quad \mathrm{~d}_{65}=\frac{\left(\mathrm{d}_{60} \mathrm{~d}_{63}-\mathrm{d}_{61} \mathrm{~d}_{62}\right)}{\mathrm{d}_{60}^{2}+\mathrm{d}_{61}^{2}}
$$$$
\mathrm{l}_{1}=\frac{\mathrm{s}_{15}^{2}}{4}-\frac{\mathrm{s}_{16}^{2}}{4} \quad, \quad \mathrm{l}_{2}=\mathrm{s}_{15}\left(\frac{\mathrm{s}_{16}}{2}\right)
$$$$
\mathrm{l}_{3}=\left(\mathrm{d}_{64} \mathrm{l}_{1}+\mathrm{d}_{65} \mathrm{l}_{2}\right) \quad, \quad \mathrm{l}_{4}=\left(\mathrm{d}_{65} \mathrm{l}_{1}-\mathrm{d}_{64} \mathrm{l}_{2}\right)
$$$$
1_{5}=A_{13}^{2}-B_{10}^{2}
$$ 
$1_{6}=2 \mathrm{~A}_{13} \mathrm{~B}_{10}$

$\mathrm{l}_{7}=\left(-\frac{\mathrm{h}_{1}}{2} \mathrm{~d}_{14}-1\right) \quad, \quad \mathrm{l}_{8}=\frac{\mathrm{h}_{1} \mathrm{~d}_{15}}{2}$

$\mathrm{d}_{14}=\left(\beta_{1}+\sqrt{\frac{\beta_{1} \sqrt{\beta_{1}^{2}+\mathrm{n}^{2}}+\beta_{1}^{2}}{2}}\right) \quad, \quad \mathrm{d}_{15}=\sqrt{\frac{\beta_{1} \sqrt{\beta_{1}^{2}+\mathrm{n}^{2}}-\beta_{1}^{2}}{2}}$

$1_{9}=\left(1-\frac{\mathrm{h}_{1} \mathrm{~s}_{15}}{2}\right) \quad, \quad \mathrm{l}_{10}=\frac{\mathrm{h}_{1} \mathrm{~s}_{16}}{2}$

$1_{11}=\left(\mathrm{A}_{12} 1_{9}-\mathrm{B}_{9} 1_{10}\right)-2 \alpha_{1}\left(1_{5} 1_{9}-1_{6} 1_{10}\right)-2 \alpha_{1}\left(1_{1} 1_{7}-1_{2} 1_{8}\right)$

$1_{12}=\left(B_{9} 1_{9}+A_{12} 1_{10}\right)-2 \alpha_{1}\left(1_{6} 1_{9}+1_{5} 1_{10}\right)+2 \alpha_{1}\left(1_{2} 1_{7}+1_{1} 1_{8}\right)$

$1_{13}=\left(A_{12} 1_{9}+B_{9} 1_{10}\right) \quad, \quad 1_{14}=\left(B_{9} 1_{9}+A_{12} 1_{10}\right)$

$\mathrm{d}_{1}=\mathrm{A}_{13} \mathrm{l}_{13}+\mathrm{l}_{14} \mathrm{~B}_{10} \quad, \quad \mathrm{~d}_{2}=\mathrm{A}_{13} \mathrm{l}_{14}-\mathrm{B}_{10} \mathrm{l}_{13}$

$\mathrm{d}_{3}=\mathrm{A}_{13}^{2} \mathrm{l}_{11}+\mathrm{B}_{10}^{2} \mathrm{l}_{11} \quad, \quad \mathrm{l}_{15}=\mathrm{A}_{13}^{2} \mathrm{l}_{12}+\mathrm{B}_{10}^{2} \mathrm{l}_{12}$

$l_{22}=\frac{\mathrm{d}_{1} \mathrm{~d}_{3}+\mathrm{d}_{2} \mathrm{l}_{15}}{\mathrm{~d}_{3}^{2}+\mathrm{l}_{15}^{2}} \quad, \quad \mathrm{l}_{23}=\frac{\mathrm{d}_{2} \mathrm{~d}_{3}-\mathrm{d}_{1} \mathrm{l}_{15}}{\mathrm{~d}_{3}^{2}+\mathrm{l}_{15}^{2}}$

$d_{4}=\left\{-l_{3}-A_{15}-A_{16}-\frac{x_{3}^{2}\left(A_{4} A_{5}+B_{4} B_{5}\right)}{A_{5}^{2}+B_{5}^{2}}-\frac{S^{2}\left(A_{6} A_{7}+B_{6} B_{5}\right)}{A_{7}^{2}+B_{5}^{2}}-\frac{\beta_{1}^{2}\left(A_{8} A_{9}-B_{7} B_{5}\right)}{A_{9}^{2}+B_{5}^{2}}\right\}$

$d_{5}=\left\{-l_{4}-B_{12}-B_{13}-\frac{x_{3}^{2}\left(A_{4} B_{5}-A_{5} B_{4}\right)}{A_{5}^{2}+B_{5}^{2}}-\frac{S^{2}\left(A_{6} B_{5}-A_{7} B_{6}\right)}{A_{7}^{2}+B_{5}^{2}}\right.$

$\left.-\frac{\beta_{1}^{2}\left(\mathrm{~A}_{8} \mathrm{~B}_{5}+\mathrm{A}_{9} \mathrm{~B}_{7}\right)}{\mathrm{A}_{9}^{2}+\mathrm{B}_{5}^{2}}-\mathrm{A}_{10}+\frac{4 \mathrm{u}_{0}^{\prime \prime}(0) \beta_{1}}{\mathrm{n}}\right\}$

$\mathrm{d}_{6}=\left(\mathrm{l}_{22} \mathrm{~d}_{4}-\mathrm{l}_{23} \mathrm{~d}_{5}\right) \quad, \quad \mathrm{d}_{7}=\left(\mathrm{l}_{23} \mathrm{~d}_{4}+\mathrm{l}_{22} \mathrm{~d}_{5}\right)$

$\mathrm{d}_{8}=-\beta_{1}-\sqrt{\frac{\beta_{1} \sqrt{\beta_{1}^{2}+\mathrm{n}^{2}}+\beta_{1}^{2}}{2}} \quad, \quad \mathrm{~d}_{9}=-\sqrt{\frac{\beta_{1} \sqrt{\beta_{1}^{2}+\mathrm{n}^{2}}-\beta_{1}^{2}}{2}}$

$\mathrm{L}_{3}=\frac{\mathrm{d}_{9} \mathrm{y}}{2}, \mathrm{C}_{1}=\cos \mathrm{L}_{3}, \mathrm{C}_{2}=\sin \mathrm{L}_{3}$

$\mathrm{L}_{2}=-\frac{\mathrm{s}_{16} \mathrm{y}}{2}, \mathrm{C}_{7}=\cos \mathrm{L}_{2}, \mathrm{C}_{8}=\sin \mathrm{L}_{2}$

$\mathrm{d}_{66}=\mathrm{e}^{\mathrm{s}_{15} \mathrm{y} / 2}\left(\mathrm{~d}_{64} \mathrm{C}_{7}-\mathrm{d}_{65} \mathrm{C}_{8}\right) \quad, \quad \mathrm{d}_{67}=\mathrm{e}^{\mathrm{s}_{15} \mathrm{y} / 2}\left(\mathrm{~d}_{65} \mathrm{C}_{7}+\mathrm{d}_{64} \mathrm{C}_{8}\right)$

$\mathrm{d}_{10}=\left(-\operatorname{Pr}-\sqrt{\frac{\sqrt{\left(\operatorname{Pr}^{2}+4 \mathrm{R}\right)^{2}+\operatorname{Pr}^{2} \mathrm{n}^{2}}+\left(\operatorname{Pr}^{2}+4 \mathrm{R}\right)}{2}}\right)$

$d_{11}=\left(-\sqrt{\frac{\sqrt{\left(\operatorname{Pr}^{2}+4 R\right)^{2}+\operatorname{Pr}^{2} n^{2}}-\left(\operatorname{Pr}^{2}+4 R\right)}{2}}\right)$ 


$$
\begin{aligned}
& \mathrm{L}_{4}=\frac{\mathrm{d}_{11} \mathrm{y}}{2}, \mathrm{C}_{3}=\cos \mathrm{L}_{4}, \mathrm{C}_{4}=\sin \mathrm{L}_{4} \\
& \mathrm{~d}_{16}=\mathrm{e}^{\mathrm{d}_{10} \mathrm{y} / 2}\left(\mathrm{~s}_{1} \mathrm{C}_{3}+\mathrm{s}_{2} \mathrm{C}_{4}\right) \\
& \mathrm{d}_{17}=\mathrm{e}^{\mathrm{d}_{10} \mathrm{y} / 2}\left(\mathrm{~s}_{1} \mathrm{C}_{4}-\mathrm{s}_{2} \mathrm{C}_{3}\right) \\
& d_{12}=-S c-\sqrt{\frac{S c \sqrt{{S c^{2}+n^{2}}^{2}+S^{2}}}{2}}, d_{13}=-\sqrt{\frac{S c \sqrt{{S c^{2}+n^{2}}^{2}-S^{2}}}{2}} \\
& \mathrm{~L}_{5}=\frac{\mathrm{d}_{13} \mathrm{y}}{2}, \mathrm{C}_{5}=\cos \mathrm{L}_{5}, \mathrm{C}_{6}=\sin \mathrm{L}_{5} \\
& \mathrm{~d}_{18}=\mathrm{e}^{\mathrm{d}_{12 \mathrm{y}} / 2}\left(\mathrm{~s}_{3} \mathrm{C}_{5}-\mathrm{s}_{4} \mathrm{C}_{6}\right), \quad \mathrm{d}_{19}=\mathrm{e}^{\mathrm{d}_{22} \mathrm{y} / 2}\left(\mathrm{~s}_{4} \mathrm{C}_{5}+\mathrm{s}_{3} \mathrm{C}_{6}\right) \\
& l_{24}=\left(-\frac{\mathrm{d}_{6} \mathrm{~d}_{8}}{2}+\frac{\mathrm{d}_{7} \mathrm{~d}_{9}}{2}\right) \quad, \quad \mathrm{l}_{25}=\left(-\frac{\mathrm{d}_{6} \mathrm{~d}_{9}}{2}-\frac{\mathrm{d}_{7} \mathrm{~d}_{8}}{2}\right) \\
& 1_{26}=1_{24} \mathrm{~A}_{12}+\mathrm{l}_{25} \mathrm{~B}_{9} \quad, \quad \mathrm{l}_{27}=\left(\mathrm{A}_{12} \mathrm{l}_{25}-\mathrm{B}_{9} \mathrm{l}_{24}\right) \\
& l_{28}=\frac{1_{26}}{A_{12}^{2}+B_{9}^{2}}, l_{29}=\frac{1_{27}}{A_{12}^{2}+B_{9}^{2}} \\
& \mathrm{~d}_{20}=\mathrm{e}^{\mathrm{d}_{8} \mathrm{y} / 2}\left(\mathrm{l}_{28} \mathrm{C}_{1}-\mathrm{l}_{29} \mathrm{C}_{2}\right) \quad, \quad \mathrm{d}_{21}=\mathrm{e}^{\mathrm{d}_{8} \mathrm{y} / 2}\left(\mathrm{l}_{29} \mathrm{C}_{1}+\mathrm{l}_{28} \mathrm{C}_{2}\right) \\
& \mathrm{d}_{22}=\mathrm{s}_{7} \mathrm{e}^{\mathrm{x}_{3} \mathrm{y}} \quad, \quad \mathrm{d}_{23}=\mathrm{s}_{8} \mathrm{e}^{\mathrm{x}_{3} \mathrm{y}} \\
& \mathrm{d}_{24}=\mathrm{s}_{9} \overline{\mathrm{e}}^{\text {scy }}, \mathrm{d}_{25}=\mathrm{s}_{10} \overline{\mathrm{e}}^{\text {Scy }} \\
& \mathrm{d}_{26}=\mathrm{s}_{11} \overline{\mathrm{e}}^{\beta_{1} \mathrm{y}}, \quad \mathrm{d}_{27}=\mathrm{s}_{12} \overline{\mathrm{e}}^{\beta_{1} \mathrm{y}} \\
& \mathrm{d}_{28}=\frac{4 \mathrm{~b}_{4} \mathrm{~m}_{7}}{\mathrm{n}} \mathrm{e}^{\mathrm{x}_{9} \mathrm{y}} \\
& d_{30}=-\left(\frac{\operatorname{Pr} n}{4}\right) \sqrt{\frac{\sqrt{\left(\operatorname{Pr}^{2}+4 r\right)^{2}+\operatorname{Pr}^{2} n^{2}}-\left(\operatorname{Pr}^{2}+4 R\right)}{2}} \\
& \mathrm{~d}_{31}=\left(\frac{\operatorname{Prn}}{4}\right)\left(\frac{\operatorname{Pr}}{2}+\frac{1}{2} \sqrt{\frac{\sqrt{\left(\operatorname{Pr}^{2}+4 \mathrm{R}\right)^{2}+\operatorname{Pr}^{2} \mathrm{n}^{2}}+\left(\operatorname{Pr}^{2}+4 \mathrm{R}\right)}{2}}\right. \\
& \mathrm{l}_{30}=\frac{-\mathrm{Ax}_{3} \mathrm{~d}_{30}}{\mathrm{~d}_{30}^{2}+\mathrm{d}_{31}^{2}} \quad, \quad \mathrm{l}_{31}=\frac{\mathrm{Ax}_{3} \mathrm{~d}_{31}}{\mathrm{~d}_{30}^{2}+\mathrm{d}_{31}^{2}} \\
& \mathrm{~d}_{34}=\mathrm{e}^{\mathrm{d}_{10} \mathrm{y} / 2}\left\{\mathrm{l}_{30} \mathrm{C}_{3}-\mathrm{l}_{31} \mathrm{C}_{4}\right\} \\
& \mathrm{d}_{35}=\mathrm{e}^{\mathrm{d}_{10 \mathrm{O}} / 2}\left\{\mathrm{l}_{31} \mathrm{C}_{3}+\mathrm{l}_{30} \mathrm{C}_{4}\right\} \\
& 1_{32}=\frac{\mathrm{AA}_{3}}{\mathrm{~A}_{3}^{2}+\mathrm{B}_{3}^{2}} \quad, \quad 1_{33}=\frac{\mathrm{AB}_{3}}{\mathrm{~A}_{3}^{2}+\mathrm{B}_{3}^{2}} \\
& \mathrm{~d}_{36}=\mathrm{l}_{32} \mathrm{e}^{\mathrm{x}_{3} \mathrm{y}} \quad, \quad \mathrm{d}_{37}=\mathrm{l}_{33} \mathrm{e}^{\mathrm{x}_{3} \mathrm{y}} \\
& \mathrm{d}_{38}=-\left(\frac{\mathrm{Scn}}{4}\right)\left(\frac{1}{2} \sqrt{\frac{\mathrm{Sc} \sqrt{\mathrm{Sc}^{2}+\mathrm{n}^{2}}-\mathrm{Sc}^{2}}{2}}\right)
\end{aligned}
$$


$\mathrm{d}_{39}=\left(\frac{\mathrm{Scn}}{4}\right)\left(\frac{\mathrm{Sc}}{2}+\frac{1}{2} \sqrt{\frac{\mathrm{Sc} \sqrt{\mathrm{Sc}^{2}+\mathrm{n}^{2}}+\mathrm{Sc}^{2}}{2}}\right.$

$1_{34}=\frac{\operatorname{ASc~d}_{38}}{d_{30}^{2}+d_{39}^{2}} \quad, \quad 1_{35}=\frac{\operatorname{ASc~d}_{39}}{d_{30}^{2}+d_{39}^{2}}$

$\mathrm{C}_{5}=\cos \frac{\mathrm{d}_{13} \mathrm{y}}{2} \quad, \quad \mathrm{C}_{6}=\sin \frac{\mathrm{d}_{13} \mathrm{y}}{2}$

$\mathrm{d}_{42}=\mathrm{e}^{\mathrm{d}_{12 \mathrm{y}} / 2}\left(\mathrm{l}_{34} \mathrm{C}_{5}+\mathrm{l}_{35} \mathrm{C}_{6}\right)$

$\mathrm{d}_{43}=\mathrm{e}^{\mathrm{d}_{12} \mathrm{y} / 2}\left(1_{34} \mathrm{C}_{6}-1_{35} \mathrm{C}_{5}\right)$

$\mathrm{d}_{44}=\frac{4 \mathrm{~A}}{\mathrm{Sc} \mathrm{n}} \mathrm{e}^{-\mathrm{Scy}}$

$\mathrm{t}_{1}=\left(\mathrm{d}_{64} \frac{\mathrm{s}_{15}}{2}+\mathrm{d}_{65} \frac{\mathrm{s}_{16}}{2}\right) \quad, \quad \mathrm{t}_{2}=\left(\mathrm{d}_{65} \frac{\mathrm{s}_{15}}{2}-\mathrm{d}_{64} \frac{\mathrm{s}_{16}}{2}\right)$

$\mathrm{t}_{3}=\left\{\mathrm{s}_{1}\left(-\frac{\operatorname{Pr}}{2}-\frac{1}{2} \sqrt{\frac{\sqrt{\left(\operatorname{Pr}^{2}+4 \mathrm{R}\right)^{2}+\operatorname{Pr}^{2} \mathrm{n}^{2}}+\left(\operatorname{Pr}^{2}+4 \mathrm{R}\right)}{2}}\right)\right.$

$\left.-\mathrm{S}_{2}\left(\frac{1}{2} \sqrt{\frac{\sqrt{\left(\operatorname{Pr}^{2}+4 \mathrm{R}\right)^{2}+\operatorname{Pr}^{2} \mathrm{n}^{2}}-\left(\operatorname{Pr}^{2}+4 \mathrm{R}\right)}{2}}\right)\right\}$

$\mathrm{t}_{4}=\left\{-\mathrm{s}_{2}\left(-\frac{\operatorname{Pr}}{2}-\frac{1}{2} \sqrt{\frac{\sqrt{\left(\operatorname{Pr}^{2}+4 \mathrm{R}\right)^{2}+\operatorname{Pr}^{2} \mathrm{n}^{2}}+\left(\operatorname{Pr}^{2}+4 \mathrm{R}\right)}{2}}\right)\right.$

$\left.-\mathrm{S}_{1}\left(\frac{1}{2} \sqrt{\frac{\sqrt{\left(\mathrm{Pr}^{2}+4 \mathrm{R}\right)^{2}+\mathrm{Pr}^{2} \mathrm{n}^{2}}-\left(\mathrm{Pr}^{2}+4 \mathrm{R}\right)}{2}}\right)\right\}$

$\mathrm{t}_{5}=\left\{\mathrm{s}_{3}\left(-\frac{\mathrm{Sc}}{2}-\frac{1}{2} \sqrt{\frac{\mathrm{Sc} \sqrt{\mathrm{Sc}^{2}+\mathrm{n}^{2}}+\mathrm{Sc}^{2}}{2}}\right)+\mathrm{s}_{4}\left(\frac{1}{2} \sqrt{\frac{\mathrm{Sc} \sqrt{\mathrm{Sc}^{2}+\mathrm{n}^{2}}-\mathrm{Sc}^{2}}{2}}\right)\right\}$

$\mathrm{t}_{6}=\left\{\mathrm{s}_{4}\left(-\frac{\mathrm{Sc}}{2}-\frac{1}{2} \sqrt{\frac{\mathrm{Sc} \sqrt{\mathrm{Sc}^{2}+\mathrm{n}^{2}}+\mathrm{Sc}^{2}}{2}}\right)-\mathrm{s}_{3}\left(\frac{1}{2} \sqrt{\frac{\mathrm{Sc} \sqrt{\mathrm{Sc}^{2}+\mathrm{n}^{2}}-\mathrm{Sc}^{2}}{2}}\right)\right\}$

$\mathrm{t}_{7}=\left\{\mathrm{l}_{28}\left(-\frac{\beta_{1}}{2}-\frac{1}{2} \sqrt{\frac{\beta_{1} \sqrt{\beta_{1}^{2}+\mathrm{n}^{2}}+\beta_{1}^{2}}{2}}\right)+\mathrm{l}_{29}\left(\frac{1}{2} \sqrt{\frac{\beta_{1} \sqrt{\beta_{1}^{2}+\mathrm{n}^{2}}-\beta_{1}^{2}}{2}}\right)\right\}$ 


$$
\begin{aligned}
& \mathrm{t}_{8}=\left\{1_{29}\left(-\frac{\beta_{1}}{2}-\frac{1}{2} \sqrt{\frac{\beta_{1} \sqrt{\beta_{1}^{2}+\mathrm{n}^{2}}+\beta_{1}^{2}}{2}}\right)-\mathrm{l}_{28}\left(\frac{1}{2} \sqrt{\frac{\beta_{1} \sqrt{\beta_{1}^{2}+\mathrm{n}^{2}}-\beta_{1}^{2}}{2}}\right)\right\} \\
& \mathrm{t}_{9}=\mathrm{s}_{7} \mathrm{x}_{3}, \quad \mathrm{t}_{10}=\mathrm{s}_{8} \mathrm{x}_{3} \\
& \mathrm{t}_{11}=\mathrm{s}_{9} \mathrm{Sc} \quad, \quad \mathrm{t}_{12}=\mathrm{s}_{10} \mathrm{Sc} \\
& \mathrm{t}_{13}=\mathrm{s}_{11} \beta_{1} \quad, \quad \mathrm{t}_{14}=\mathrm{s}_{12} \beta_{1} \\
& \mathrm{t}_{15}=\mathrm{d}_{58} \mathrm{x}_{9} \quad, \quad \mathrm{t}_{16}=\left(\mathrm{t}_{1}+\mathrm{t}_{3}+\mathrm{t}_{5}+\mathrm{t}_{7}+\mathrm{t}_{9}-\mathrm{t}_{11}-\mathrm{t}_{13}\right) \\
& \mathrm{t}_{17}=\left(\mathrm{t}_{2}+\mathrm{t}_{4}+\mathrm{t}_{6}+\mathrm{t}_{8}+\mathrm{t}_{10}+\mathrm{t}_{12}-\mathrm{t}_{14}+\mathrm{t}_{15}\right) \\
& \mathrm{t}_{18}=\left(\mathrm{l}_{30}+\mathrm{l}_{32}\right) \quad, \quad \mathrm{t}_{19}=\left(\mathrm{l}_{31}+\mathrm{l}_{33}\right) \\
& \mathrm{t}_{20}=\mathrm{m}_{3}+\in\left(\mathrm{t}_{18} \cos \mathrm{nt}-\mathrm{t}_{19} \sin \mathrm{nt}\right) \\
& \mathrm{t}_{21}=\left(\mathrm{t}_{18} \sin \mathrm{nt}+\mathrm{t}_{19} \cos \mathrm{nt}\right)
\end{aligned}
$$

\section{Result and Discussion}

In order to get a physical insight of the problem, calculations have been made for velocity (u), angular velocity $(\square)$, temperature $(\square)$, concentration $(C)$, skin friction $(€)$ and Nusselt number $(\mathrm{Nu})$ for different values of $\mathrm{K}$ (permeability parameter), $\mathrm{M}$ (magnetic parameter), Gr (thermal Grashof number), Gc (mass Grashof number), $\mathrm{h}_{1}$ (velocity slip parameter), $\mathrm{R}$ (radiation parameter) etc. Observations are made for both the basic fluids, air $(\operatorname{Pr}=0.71, \mathrm{Sc}=0.22)$ and water $(\operatorname{Pr}=7, \mathrm{Sc}=0.61)$ fixing $\mathrm{n}=3, \mathrm{nt}=\square / 2$, $\square=0.01$.

The velocity profiles are plotted against $y$ in figures 1,2 and 3 taking $\mathrm{B}=0.5$. We observe that for both the basic fluids air and water, velocity increases with increase in $\mathrm{K}, \mathrm{Gr}, \mathrm{Gc}$ and $\square_{1}$ where as on increasing $\mathrm{M}$ and $\square_{1}$ velocity drops. On negative of radiation i.e, when absorption takes place, it tends the velocity to rise. It is note worthy, that on decreasing the velocity slip parameter $\left(h_{1}\right)$, the velocity drops at the plate but rises as we move away from the plate. On the other hand when we decrease $\mathrm{A}$, velocity increases at the plate and drops later. For the case of free flow with no slip at the boundary i.e $(\mathrm{K}=\square$, $\left.\mathrm{h}_{1}=0\right)$ as compared to $\left(\mathrm{K} \neq \infty, \mathrm{h}_{1} \neq 0\right)$ velocity drops at the plate but increases as soon as we move away from the plate. The effect of $\mathrm{K}$ can be understood physically as, when $\mathrm{K}$ increases, that is, the medium is more porous, so more fluid can flow through hence increasing the velocity of the fluid. On the whole we observe that velocity is less for water as compared to the case for air, this is because air is lighter than water.

In figure 4 , angular velocity profiles are plotted against y fixing $\mathrm{A}=\mathrm{B}=0.5, \mathrm{R}=0.05, \mathrm{Gr}=0.3, \mathrm{Gc}=$ 0.1 and $\mathrm{M}=2$. From the figure it is observed that for both the basic fluids air and water, on increasing $\mathrm{K}$ and $\square_{1}$, angular velocity decreases but, angular velocity increases with increase in $h_{1}$ and $\square_{1}$, for the case of free flow, with no slip at the boundary $\left(\mathrm{K}=\square, \mathrm{h}_{1}=0\right)$, angular velocity drops, as compared to the case of $\left(K \neq \infty, h_{1} \neq 0\right)$. In general we notice that angular velocity is lower for air as compared to water.

Temperature profiles are plotted against $y$, in figure 5. We observe for both the basic fluids air and water increase in radiation, decreases the temperature and negative of radiation i.e absorption tends the temperature to rise as when absorption of heat takes place the temperature of the fluid will rise. But the effect of $\mathrm{A}$ is different for the case of air as compared to water, since for air, when suction velocity is constant $(\mathrm{A}=0)$, the temperature rises, where as, for the same temperature drops for water.

In figure 6, concentration profiles are plotted against $\mathrm{y}$, for different values of Schmidt number (Sc), chosen in such a way that they represent the diffusing chemical species of most common interest in air (for example, the values of Schmidt number for $\mathrm{H}_{2}, \mathrm{H}_{2} \mathrm{O}, \mathrm{NH}_{3}$ and propyl benzene in air is $0.22,0.61,0.78$ and 2.62 respectively. We clearly observe that as Sc increases, concentration decreases. We also notice that for constant suction velocity $(A=0)$, concentration increases slightly for both the basic fluids air and water. Moreover, for every value of $A$, concentration is much higher for air as compared to water.

An important physical parameter skin friction is plotted against $\mathrm{K}$, in figures 7 and 8 . It is noteworthy from the figures that for both the basic fluids air and water, increase in Gr, Gc and $\square_{1}$, increases the skin friction, where as decrease in $\mathrm{M}, \mathrm{h}_{1}$ and $\square_{1}$, increases the skin friction. The effect of velocity slip parameter $\left(\mathrm{h}_{1}\right)$, can be seen physically as when $h_{1}$ decreases i.e slip at the boundary is decreased, this will lead to increase in 
skin friction, which exactly happens in our case for both the basic fluids. From both the figures we observe that skin friction is higher for air as compared to water.

Another important physical parameter, Nusselt number is plotted against A in figure 9. It is observed that for both the basic fluids air and water, increase in radiation increases the Nusselt number and when absorption (negative of radiation) takes place Nusselt number drops. This is because when radiation increases, the rate of heat transfer is high thus Nusselt number increases. It is noteworthy that Nusselt number is higher for water as compared to air.

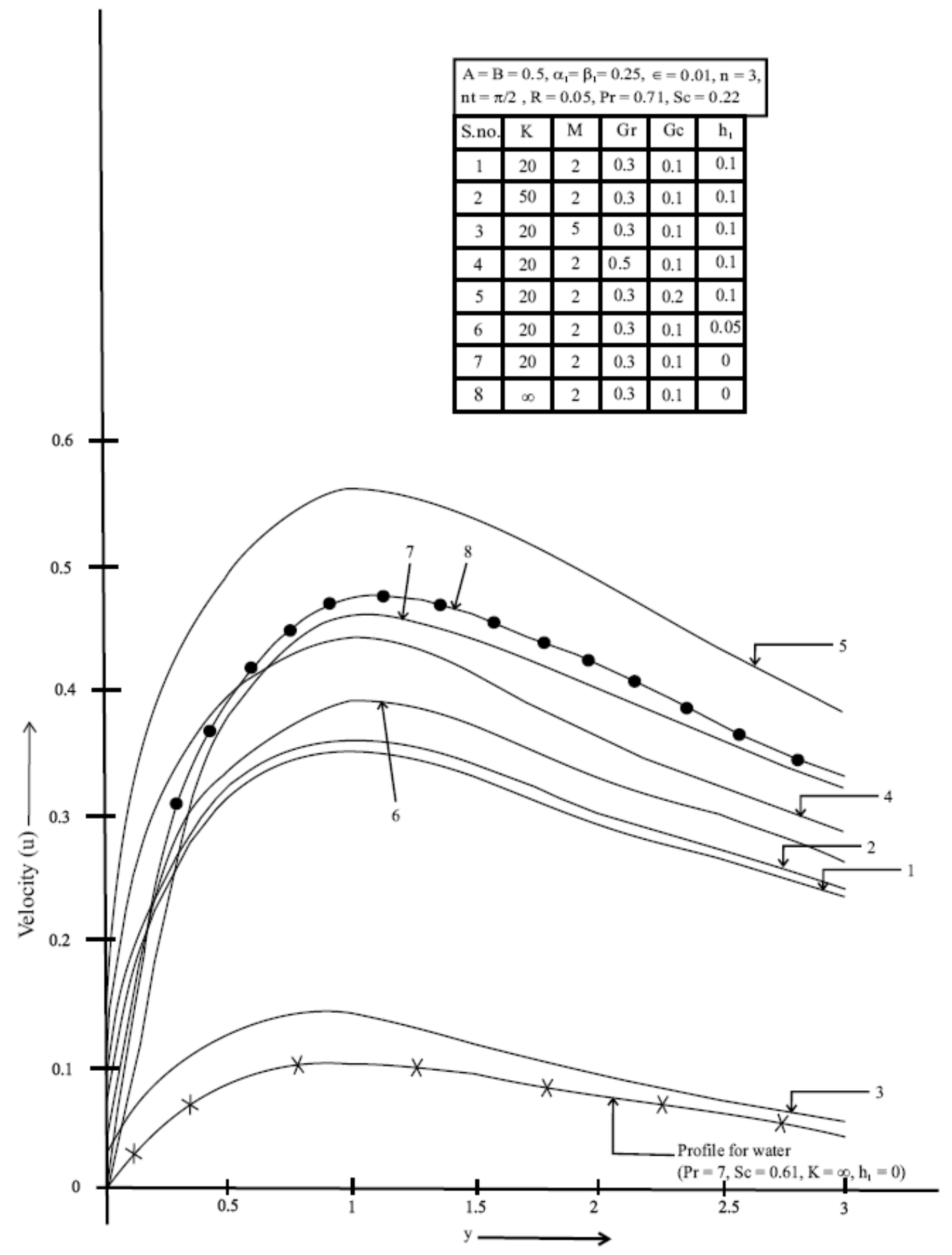

Figure 1: Velocity profiles for air plotted against y for different values of $\mathrm{K}, \mathrm{M}, \mathrm{Gr}, \mathrm{Gc}$ and $\mathrm{h}_{1}$. 


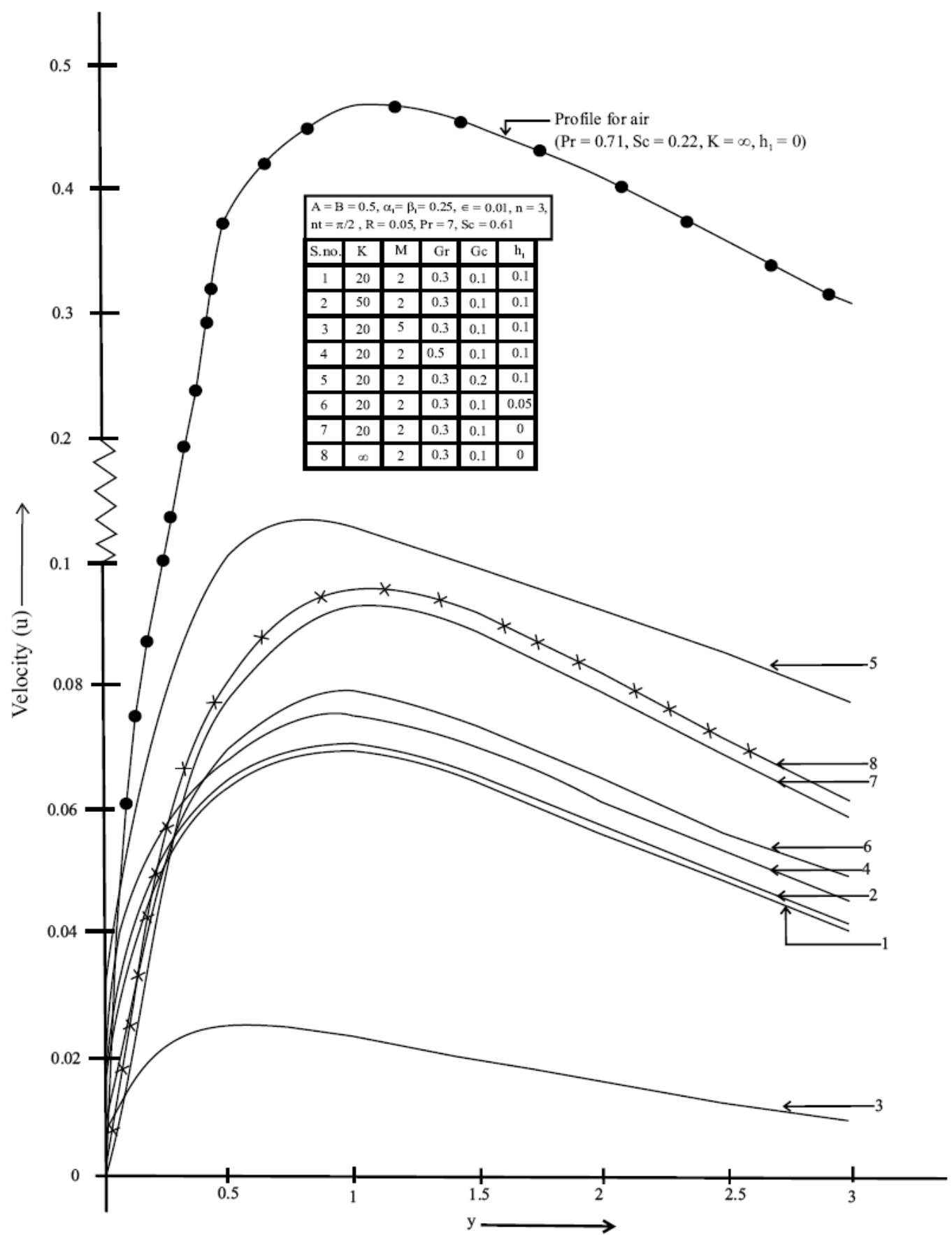

Figure 2: Velocity profiles for water plotted against y for different values of $\mathrm{K}, \mathrm{M}, \mathrm{Gr}, \mathrm{Gc}$ and $\mathrm{h}_{1}$. 


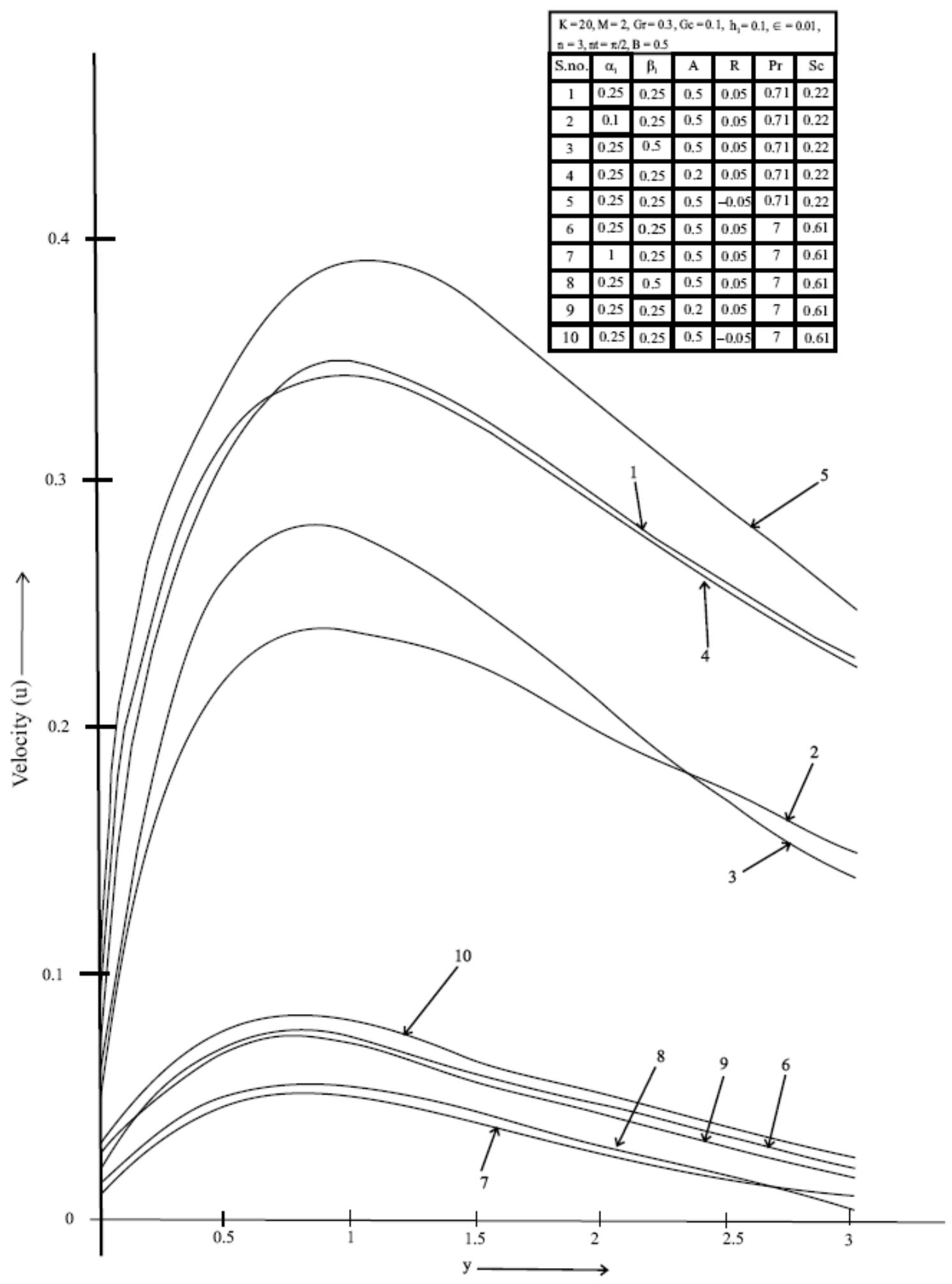

Figure 3: Velocity profiles plotted against y for different values of $\alpha_{1}, \beta_{1}, A, R, \operatorname{Pr}$ and Sc. 


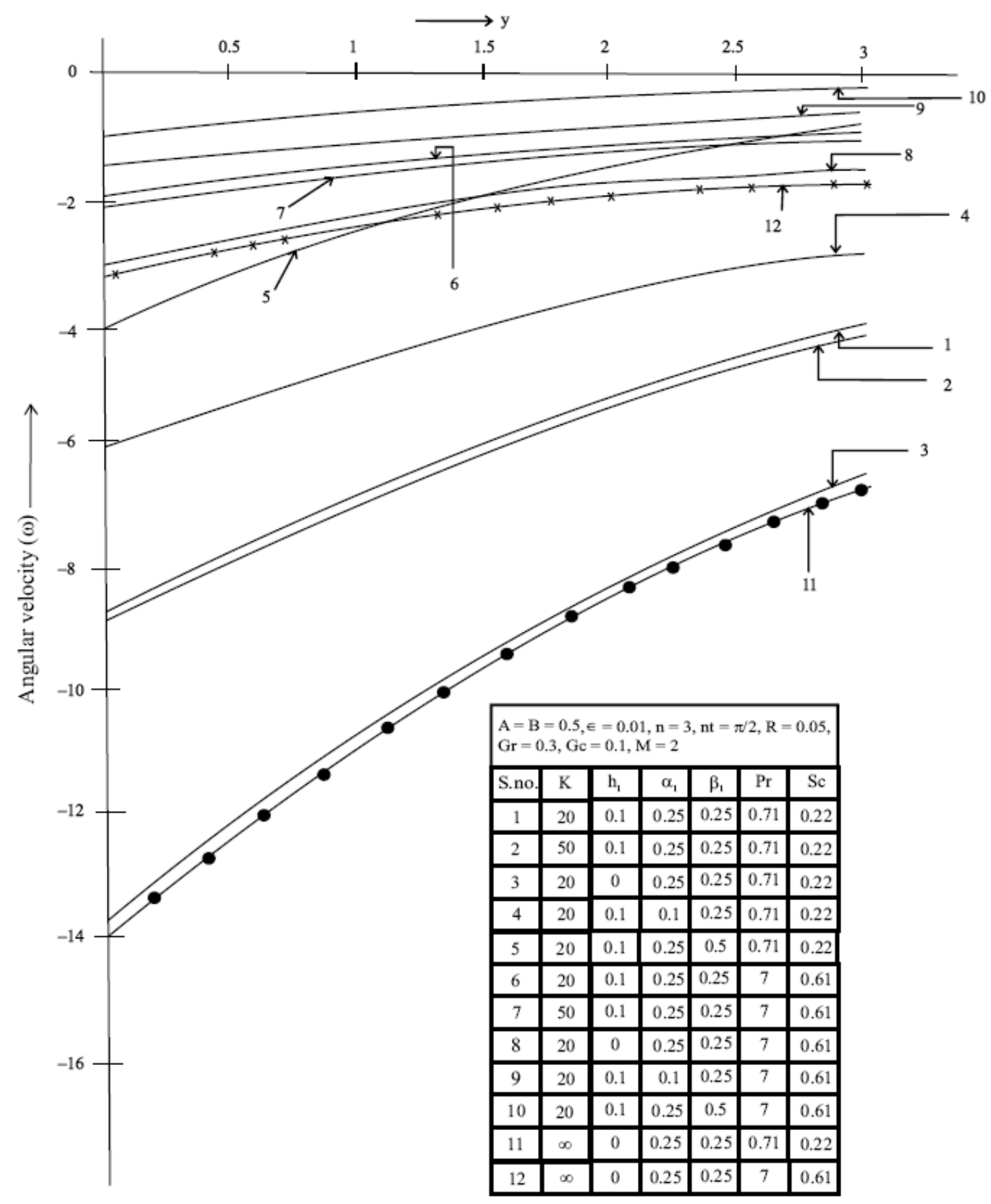

Figure 4: Angular velocity profiles plotted against y for different values of $\mathrm{K}, \mathrm{h}_{1}, \alpha_{1}, \beta_{1}, \operatorname{Pr}$ and Sc. 


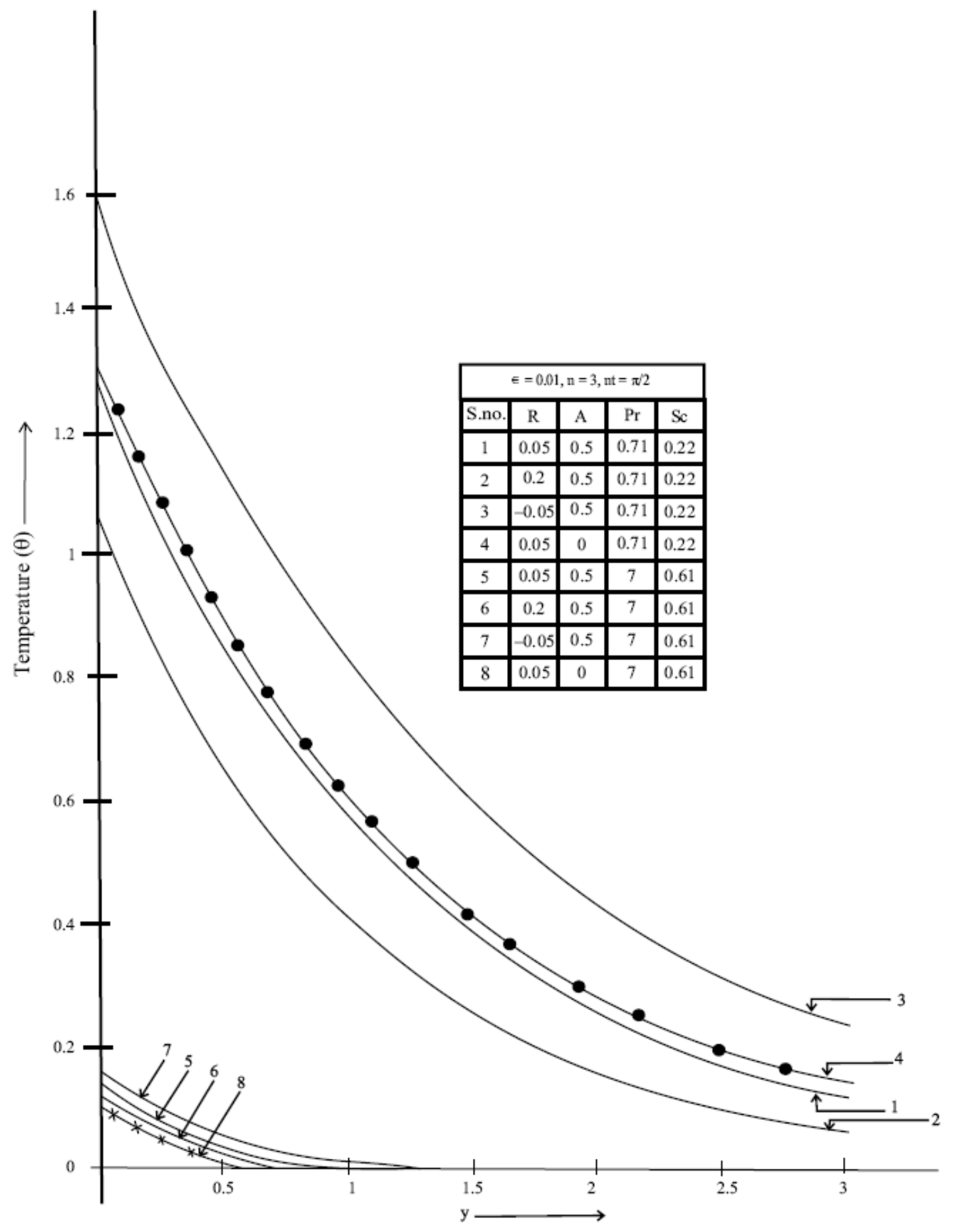

Figure 5: Temperature profiles plotted against y for different values of $\mathrm{R}, \mathrm{A}, \mathrm{Pr}$ and Sc. 


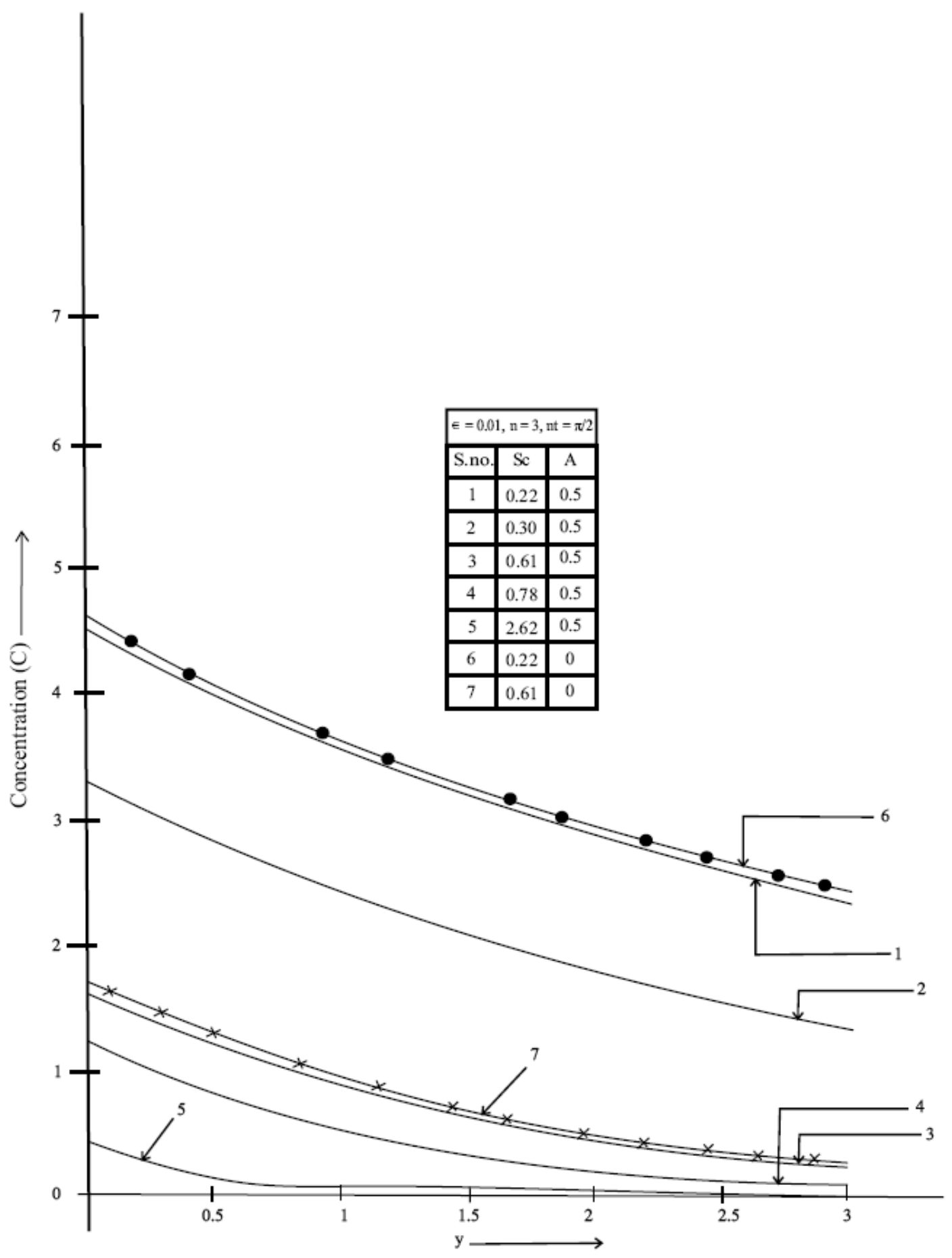

Figure 6: Concentration profiles plotted against y for different values of Sc and A. 


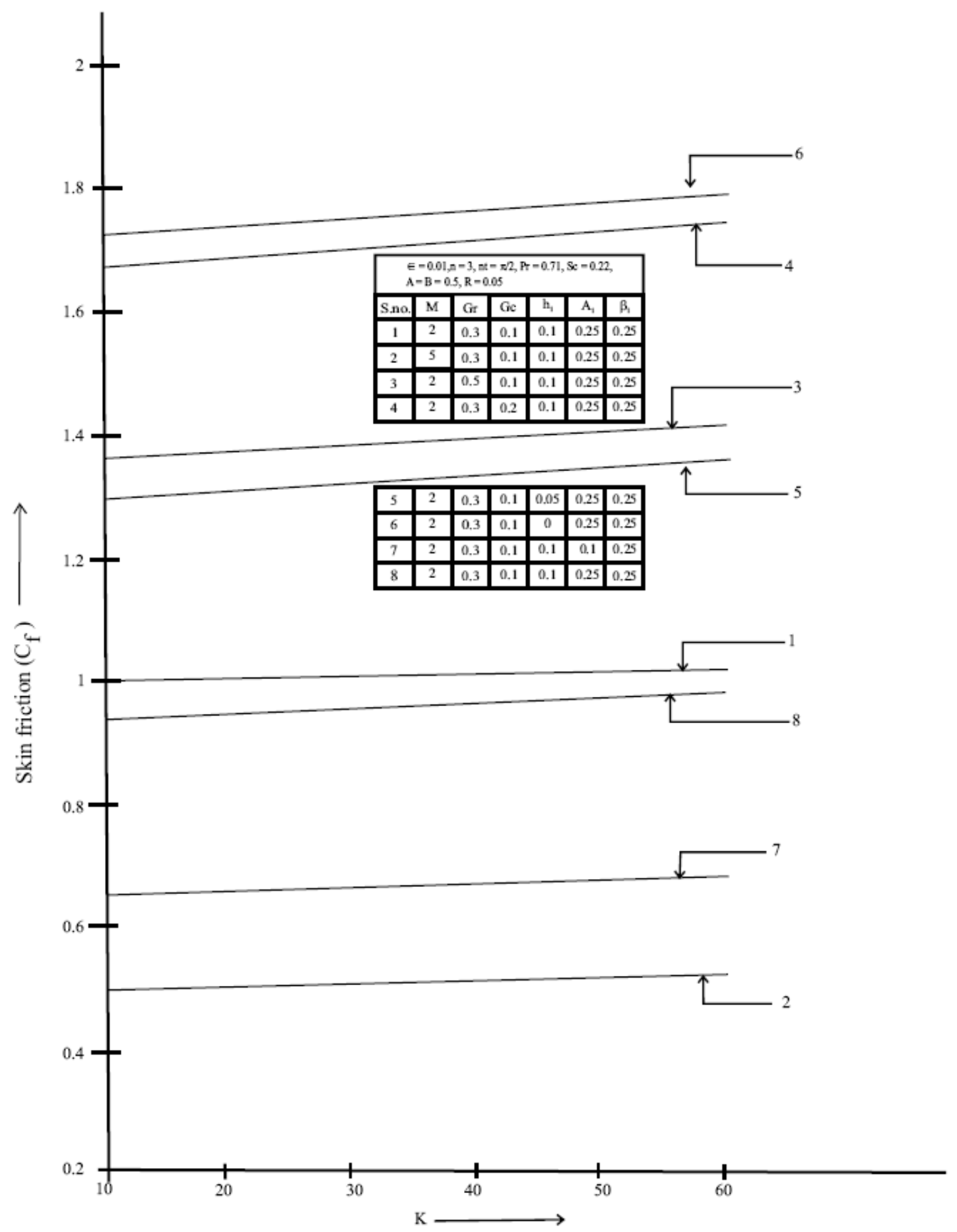

Figure 7: Skin friction for air plotted against $\mathrm{K}$ for different values of $\mathrm{M}, \mathrm{Gr}, \mathrm{Gc}, \mathrm{h}_{1}, \alpha_{1}, \beta_{1}$. 


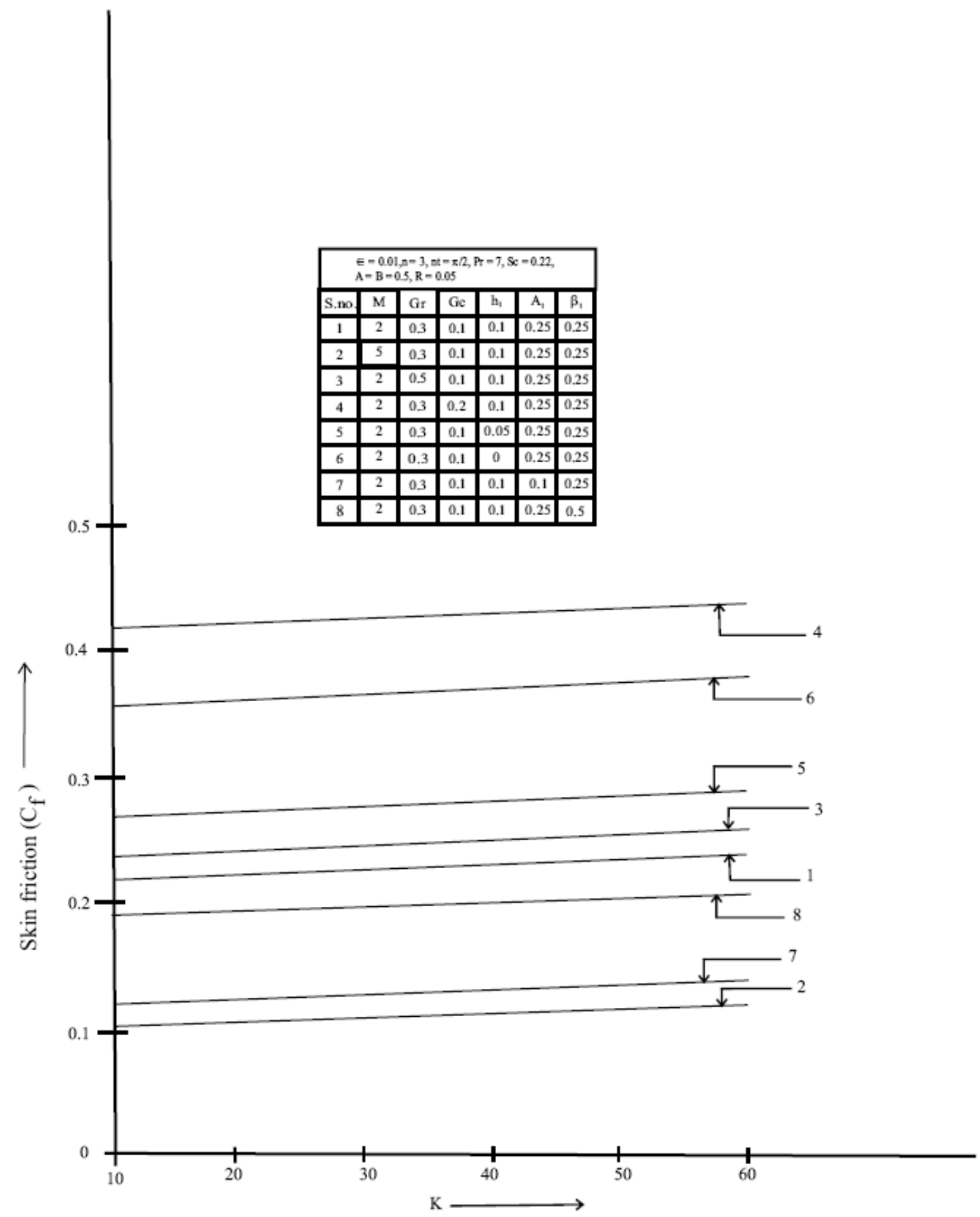

Figure 8: Skin friction for water plotted against $\mathrm{K}$ for different values of $\mathrm{M}, \mathrm{Gr}, \mathrm{Gc}, \mathrm{h}_{1}, \alpha_{1}, \beta_{1}$. 


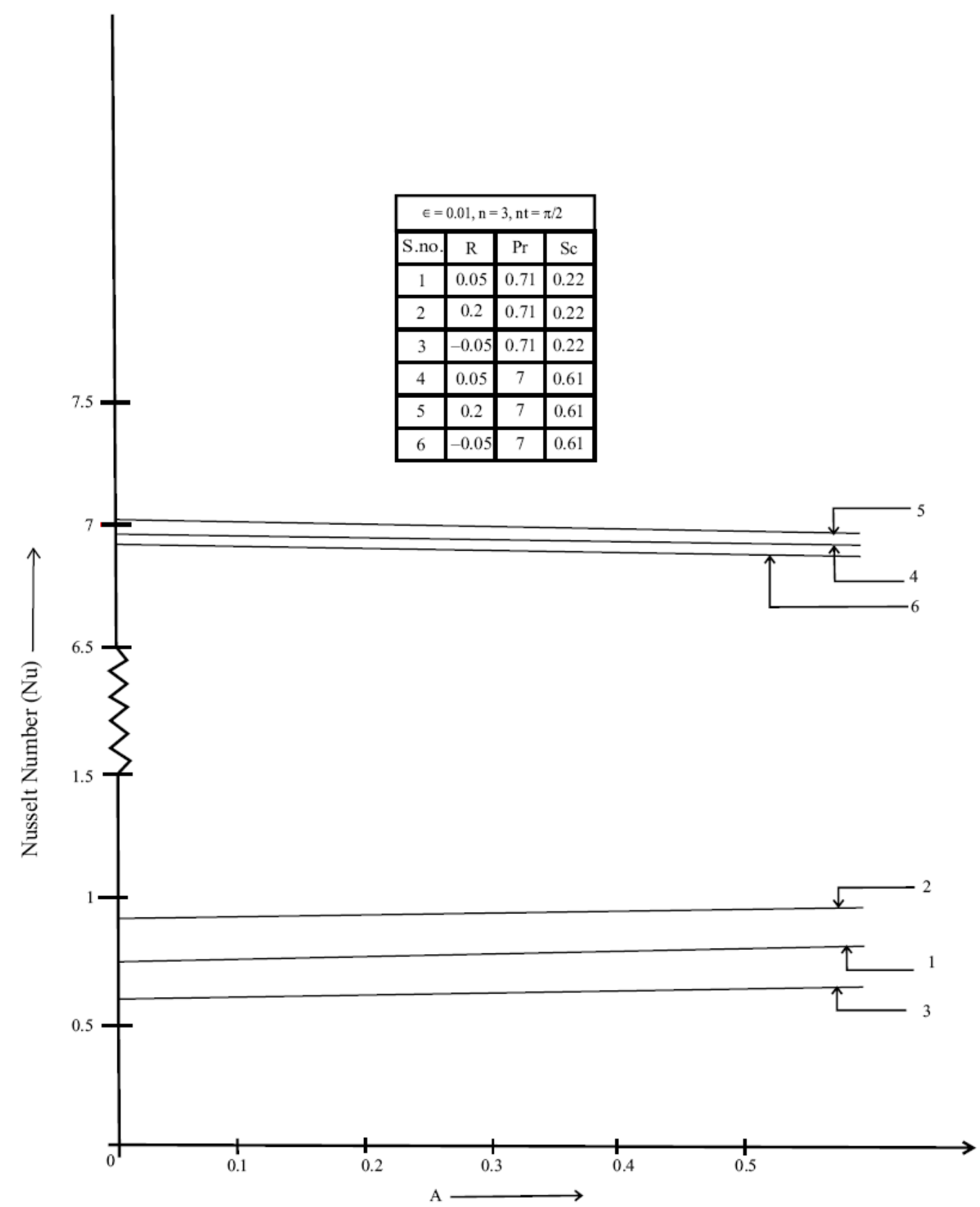

Figure 9: Nusselt number plotted against A for different values of R, Pr and Sc. 


\section{References}

[1] Das and R. Das, MHD free convection flow near a moving plate in presence of thermal radiation, An analytic solution, Moldavian Journal of Physics and Science, 8, 2009, 3-4.

[2] P. Singh and C.B. Gupta, MHD free convection flow of a viscous fluid through a porous medium bounded by an oscillating porous plate in slip flow regime with mass transfer, Indian Journal of Theoretical Physics, 53 (2), 2005, 111-120.

[3] S. Ahmed, Effects of unsteady free convection MHD flow through a porous medium bounded by an infinite vertical porous plate, Bulletin of Calcutta Mathematical Society, 99 (5), 2007, 511-522.

[4] P.K. Sahoo, N. Datta and S. Biswal, Magnetohydro dynamic unsteady free convection flow past an infinite vertical plate with constant suction and heat sink, Indian Journal of Pure and Applied Mathematics, 34 (1), 2003, 145-155.

[5] C.I. Cookey, A. Ogulu and V.M. Omubo-Pepple, Influence of viscous dissipation and radiation on unsteady MHD free convection flow past an infinite heated vertical plate in a porous medium with time dependent suction, International Journal of Heat and Mass Transfer, 46, 2003, 2305-2311.

[6] E.M. Aboeldahab and G.E.D.A. Azzam, Unsteady three dimensional combined heat and mass transfer for convective flow over a stretching surface with time dependent chemical reaction, Acta Mechenica, 184, 2006, 121-136.

[7] E. Magyari, I. Pop and B. Keller, Analytic solution for unsteady free convection in porous media, Journal of Engineering Mathematics, 48, 2004, 93-104.

[8] C. Geindreau and L. Auriault, Magnetohydrodynamic flows in porous media. Journal of fluid mechanics, 466, 2002, 343-363.

[9] S. Ahmed and N. Ahmed, Oscillatory three dimensional flow through a porous medium with viscous dissipative heat, International Journal of Applied Mathematics, 21 (3), 2008, 419-434.

[10] S. Ahmed and N. Ahmed, Two dimensional MHD Oscillatory flow along a uniformly moving infinite vertical porous plate bounded by a porous medium, Indian Journal of Pure and Applied Mathematics, 35 (12), 2004, 1309-1319.

[11] G. Lukaszewicz, Micropolar fluids, Theory and Applications, Boston, Birkhauser, (1999)

[12] Y.J. Kim, Unsteady MHD convection flow of a polar fluids past a vertical moving porous plate in a porous medium, International Journal of Heat and Mass Transfer, 44, 2001, 2791-2799.

[13] P.M. Patil and P.S. Kulkarni, Effects of Chemical reaction on free convective flow of a polar fluid through a porous medium in the presence of internal heat generation, International Journal of Thermal Science, 47, 2008, 1043-1054.

[14] N.C. Jain and P. Gupta, Effects of rotational parameter on unsteady magnetopolar free convection flow with thermal radiation in slip flow regime, Ganita Sandesh, 20, 2006, 97-110.

[15] N.C. Jain and P. Gupta, Unsteady magnetopolar free convection flow in slip flow regime with variable permeability and constant heat flux. Journal of Energy, Heat and Mass Transfer, 29, 2007, 227-240.

[16] C.V. Cheng, Natural convection heat and mass transfer from a sphere in micropolar fluids with constant wall temperature and concentration, International Communications in Heat and Mass Transfer, 35, 2008, 750-755.

[17] N.C. Jain, D. Chaudhary and R.N. Jat, Effects of rotational and couple, stress parameters on unsteady magnetopolar free convection flow with mass transfer and thermal radiation in slip flow regime, Journal of Energy, Heat and Mass Transfer, 32, 2010, 333-346.

[18] R.C. Chaudhary and P. Jain, combined heat and mass transfer in magneto-micropolar fluid flow from radiate surface with variable permeability in slip flow regime, Journal of Applied Mathematics and Mechanics, 87 (8-9), 2007.

[19] T. Fang, J. Zhang and S. Yao, Slip MHD viscous flow over a stretching sheet-an exact solution, Communications in Non linear Science and Numerical Simulation, 14 (11), 2009, 3731-3737.

[20] A. Sahin, Influence of chemical reaction on transient MHD free convective flow over a vertical plate in slip flow regime, Emirates Journal of Engineering Research, 15 (1), 2010, 25-34.

[21] W.G. England and A.F. Emery, Thermal radiation effects on the laminar free convection boundary layer of an absorbing gas, Journal of Heat Transfer, 91, 1969, 37-44. 